\title{
Regularity of the Obstacle Problem for a Fractional Power of the Laplace Operator
}

\author{
LUIS SILVESTRE \\ The University of Texas at Austin
}

\begin{abstract}
Given a function $\varphi$ and $s \in(0,1)$, we will study the solutions of the following obstacle problem:

- $u \geq \varphi$ in $\mathbb{R}^{n}$,

- $(-\triangle)^{s} u \geq 0$ in $\mathbb{R}^{n}$,

- $(-\triangle)^{s} u(x)=0$ for those $x$ such that $u(x)>\varphi(x)$,

- $\lim _{|x| \rightarrow+\infty} u(x)=0$.

We show that when $\varphi$ is $C^{1, s}$ or smoother, the solution $u$ is in the space $C^{1, \alpha}$ for every $\alpha<s$. In the case where the contact set $\{u=\varphi\}$ is convex, we prove the optimal regularity result $u \in C^{1, s}$. When $\varphi$ is only $C^{1, \beta}$ for a $\beta<s$, we prove that our solution $u$ is $C^{1, \alpha}$ for every $\alpha<\beta$. (C) 2006 Wiley Periodicals, Inc.
\end{abstract}

\section{Contents}

1. Introduction

2. Preliminary Properties of the Fractional Laplace Operator 6

3. Basic Properties of the Free Boundary Problem 17

4. An Improvement in Regularity 26

5. Towards Optimal Regularity $\quad 38$

$\begin{array}{ll}\text { Bibliography } & 46\end{array}$

\section{Introduction}

\subsection{Setup of Problem}

In this work, we will consider a function $u$ that solves an obstacle problem for the operator $(-\triangle)^{s}$ for $s \in(0,1)$. Given a continuous function $\varphi$ with a compact support (or at least rapid decay at infinity), we consider a continuous function $u$ satisfying

$$
\begin{aligned}
u \geq \varphi & \text { in } \mathbb{R}^{n}, \\
(-\triangle)^{s} u \geq 0 & \text { in } \mathbb{R}^{n},
\end{aligned}
$$

Communications on Pure and Applied Mathematics, Vol. LIX, 0001-0046 (2006)

(c) 2006 Wiley Periodicals, Inc. 


$$
\begin{aligned}
(-\triangle)^{s} u(x) & =0 \quad \text { for those } x \text { such that } u(x)>\varphi(x), \\
\lim _{|x| \rightarrow+\infty} u(x) & =0 .
\end{aligned}
$$

When $\varphi \in C^{\infty}$, the expected optimal regularity for this type of problem is $C^{1, s}$. We prove $u \in C^{1, \alpha}$ for every $\alpha<s$. In the case when the contact set $\{u=\varphi\}$ is convex, we achieve the optimal result $u \in C^{1, s}$. If $\varphi$ is only $C^{\alpha}$ for $\alpha<1$ or Lipschitz, we will prove that $u$ has the same modulus of continuity (Theorem 3.8). If $\varphi$ is $C^{1, \beta}$, we will prove that $u \in C^{1, \alpha}$ for every $\alpha<\min (\beta, s)$ (Theorem 5.8).

The existence of such a function $u$ can be obtained by variational methods as the unique minimizer of

$$
J(u):=\int_{\mathbb{R}^{n}} \int_{\mathbb{R}^{n}} \frac{|u(x)-u(y)|^{2}}{|x-y|^{n+2 s}} \mathrm{~d} x \mathrm{~d} y .
$$

from all the functions $u$ that satisfy $\varphi \leq u$ and are in a suitable function space.

We can also obtain $u$ by adapting Perron's method, as the least supersolution of $(-\triangle)^{s}$ such that $u \geq \varphi$. Another approach is by choosing the optimal closed set $\Lambda \subset \mathbb{R}^{n}$ to maximize the solution of

- $u(x)=\varphi(x)$ in $\Lambda$,

- $(-\triangle)^{s} u=0$ in $\mathbb{R}^{n} \backslash \Lambda$,

- $\lim _{|x| \rightarrow \infty} u(x)=0$.

We will choose the variational approach as the starting point. Then we will prove that $u$ also solves the other two (equivalent) problem formulations. Our main focus, however, is the regularity of the solution.

Since we will be dealing with the operators $(-\triangle)^{\sigma}$, we will need several related results. Most of the present theory can be found in [9]. We will cite some results from there, and we will prove some others when we find it useful to present them in a form more convenient to our purposes. In Section 2 we will study all the basic properties of these operators that we will need. In Section 3, we will prove the existence of a solution $u$ of our free boundary problem and prove the first regularity results. In Section 4 we will improve a regularity result; this is probably the trickiest part of the paper. Finally, in Section 5 we will present the optimal regularity result.

In the case $s=1$, our problem turns into the usual obstacle problem. Given a domain $\Omega \subset \mathbb{R}^{n}$ and a function $\varphi: \Omega \rightarrow \mathbb{R}$, in the usual obstacle problem we have a function $u$ that satisfies

- $u \geq \varphi$ in $\Omega$,

- $\Delta u \leq 0$ in $\Omega$,

- $\Delta u(x)=0$ for those $x \in \Omega$ such that $u(x)>\varphi(x)$.

The existence of this problem can be obtained by minimizing a functional in $H^{1}$ with the constraint of $u \geq \varphi$ and some given boundary condition. If $\varphi$ is a smooth function, then $u$ is expected to be more regular than just in $H^{1}(\Omega)$. In 1971 
Frehse [7] showed for the first time that $u$ is as smooth as $\phi$ up to $C^{1,1}$; another proof was given in [5]. This regularity is optimal; simple examples show that for very smooth $\phi, u$ does not get any better than $C^{1,1}$.

Most of the regularity properties of the usual obstacle problem for the Laplacian, including the regularity of the free boundary, can be found in [4].

Another related problem is the Signorini problem, or the thin-obstacle problem, where the obstacle is lower dimensional. We will continue with this problem in the next subsection.

\subsection{Applications to the Signorini Problem}

Let us consider a smooth function with rapid decay at infinity $u_{0}: \mathbb{R}^{n-1} \rightarrow \mathbb{R}$. Let $u: \mathbb{R}^{n-1} \times(0, \infty) \rightarrow \mathbb{R}$ be the unique solution of the Laplace equation in the upper half-space that vanishes at infinity with $u_{0}$ as the boundary condition:

$$
\begin{array}{ll}
u\left(x^{\prime}, 0\right)=u_{0}\left(x^{\prime}\right) & \text { for } x^{\prime} \in \mathbb{R}^{n-1}, \\
\triangle u(x)=0 & \text { for } x \in \mathbb{R}^{n-1} \times(0, \infty) .
\end{array}
$$

Consider the operator $T: u_{0}\left(x^{\prime}\right) \mapsto-\partial_{n} u\left(x^{\prime}, 0\right)$. We see that

$$
\begin{gathered}
\int_{\mathbb{R}^{n-1}} u\left(x^{\prime}, 0\right)\left(-\partial_{n} u\left(x^{\prime}, 0\right)\right) \mathrm{d} x^{\prime}=\int_{\mathbb{R}^{n-1} \times(0, \infty)}-u(x) \Delta u(x)+|\nabla u(x)|^{2} \mathrm{~d} x, \\
\int_{\mathbb{R}^{n-1} \times(0, \infty)}|\nabla u(x)|^{2} \mathrm{~d} x \geq 0 .
\end{gathered}
$$

Thus $T$ is a positive operator. Moreover, since $\partial_{n} u(x)$ is also a harmonic function, if we apply the operator twice we get

$$
T \circ T u_{0}=\left(-\partial_{n}\right)\left(-\partial_{n}\right) u\left(x^{\prime}, 0\right)=\partial_{n n} u\left(x^{\prime}, 0\right)=-\sum_{i=1}^{n-1} \partial_{i i} u\left(x^{\prime}, 0\right)=-\triangle u_{0} \text {. }
$$

Thus the operator $T$ that maps the Dirichlet-type condition $u_{0}$ into the Neumanntype $-\partial_{n} u$ is actually the operator $(-\triangle)^{1 / 2}$.

One version of the Signorini problem is this: given $\varphi$ a smooth function in $\mathbb{R}^{n-1}$, the solution $u$ of the Signorini problem is the least harmonic function in the upper half semispace $\mathbb{R}^{n-1} \times(0, \infty)$ such that $u \geq \varphi$ and $\partial_{n} u \leq 0$ on $\mathbb{R}^{n-1} \times\{0\}$. From the fact explained just above, we see that actually this problem is exactly our obstacle problem for the operator $(-\triangle)^{1 / 2}$. The regularity we obtained is therefore $C^{1,1 / 2}$ in the case $\{u=\varphi\}$ is convex, and $C^{1, \alpha}$ for every $\alpha<\frac{1}{2}$ in the general case. The optimal regularity $C^{1,1 / 2}$ was obtained very recently for this problem by Athanasopoulos and Caffarelli in [1]. The two-dimensional case was proven previously by Richardson in [12]. In 1979 Caffarelli showed $C^{1, \alpha}$-regularity for a small value of $\alpha$ in the $n$-dimensional case [3].

Usually the Signorini problem (or its equivalent formulation as the thin-obstacle problem) is studied in bounded domains. For regularity purposes, one case can be 
deduced from the other. Suppose we have a solution of the Signorini problem in a ball:

$$
\begin{aligned}
-\Delta u(x) & =0 & & \text { for }|x|<1 \text { and } x_{n}>0, \\
u(x) & =0 & & \text { for }|x|=1 \text { and } x_{n} \geq 0, \\
u\left(x^{\prime}, 0\right) & \geq \varphi\left(x^{\prime}\right) & & \text { for }\left|x^{\prime}\right| \leq 1, \\
\partial_{n} u\left(x^{\prime}, 0\right) & \leq 0 & & \text { for }\left|x^{\prime}\right| \leq 1, \\
\partial_{n} u\left(x^{\prime}, 0\right) & =0 & & \text { where } u\left(x^{\prime}, 0\right)>\varphi .
\end{aligned}
$$

For the problem to make sense, we assume that $\varphi\left(x^{\prime}\right)<0$ when $\left|x^{\prime}\right|=1$. Let $\eta$ be a radially symmetric cutoff function such that $\{\varphi>0\} \Subset\{\eta=1\}$ and supp $\eta \subset B_{1}$. The function $\eta u$ is above $\varphi$ and also satisfies $\partial_{n} \eta u\left(x^{\prime}, 0\right) \leq 0$ for $x^{\prime} \in \mathbb{R}^{n-1}$ and $\partial_{n} \eta u\left(x^{\prime}, 0\right)=0$ for those $x^{\prime} \in \mathbb{R}^{n-1}$ such that $\eta u\left(x^{\prime}, 0\right)>\varphi\left(x^{\prime}\right)$. Although $\eta u$ may not be harmonic in the upper half-space, its Laplacian is a smooth function.

Let $v$ be the unique bounded solution of the Neumann-type problem in the upper half semispace:

$$
\begin{aligned}
\Delta v(x) & =\Delta \eta u(x)=\Delta \eta(x) u(x)+2 \nabla \eta(x) \cdot \nabla u(x), \\
\partial_{n} v\left(x^{\prime}, 0\right) & =0 .
\end{aligned}
$$

Since $\triangle \eta u(x)$ is smooth and compactly supported, $v$ is a smooth function. Now $\eta u-v$ is a solution of the Signorini problem without boundary with $\varphi-v$ as the obstacle. Therefore, we reduce the regularity for the bounded case from the regularity of the unbounded case and our result applies.

\subsection{Variations of the Problem}

There are small variations of the obstacle problem that can be considered. To simplify the variational proof of existence, we could consider minimizers of the standard $H^{s}$-norm from all the functions $u$ that lie above a given obstacle $\varphi$. By the $H^{s}$-norm we mean

$$
\|u\|_{H^{s}}=\sqrt{\int_{\mathbb{R}^{n}}\left(1+|\xi|^{2 s}\right)|\hat{u}(\xi)|^{s} \mathrm{~d} \xi} .
$$

In this case we obtain a free boundary problem of the sort

- $u \geq \varphi$ in $\mathbb{R}^{n}$,

- $u+(-\triangle)^{s} u \geq 0$ in $\mathbb{R}^{n}$,

- $u+(-\triangle)^{s} u(x)=0$ for those $x$ such that $u(x)>\varphi(x)$.

The proofs of Section 3 have to be adapted to use the operator Id $+(-\triangle)^{s}$ instead of $(-\triangle)^{s}$. Once we get that the solution $u$ is semiconvex, then it is going to be Lipschitz and we can pass the term $u$ to the right-hand side; everything in Sections 4 and 5 applies without change. An advantage of this variation of the problem is that we can get existence also in the case $n=1$ and $s>\frac{1}{2}$. 
We could also consider a problem with boundary values. Let $\varphi$ be such that $\varphi(x)<0$ for every $|x| \geq 1$. Let $u$ be the minimizer of $J(u)$ (for $J$ defined in (1.5)) from all the functions $u$ that lie above $\varphi$ and $u(x)=0$ for every $x \in \mathbb{R}^{n} \backslash B_{1}$. Then we obtain the following free boundary problem:

- $u \geq \varphi$ in $\mathbb{R}^{n}$,

- $u=0$ in $\mathbb{R}^{n} \backslash B_{1}$,

- $(-\triangle)^{s} u \geq 0$ in $B_{1}$,

- $(-\triangle)^{s} u(x)=0$ for those $x \in B_{1}$ such that $u(x)>\varphi(x)$.

With a trick as in Section 1.2, our result applies to the interior regularity of this problem. However, this solution $u$ is not going to be $C^{1, \alpha}\left(\mathbb{R}^{n}\right)$ since it is not going to be differentiable across the boundary of the unit ball $\partial B_{1}$. (As a matter of fact, we cannot expect better that $C^{s}$ on $\partial B_{1}$, the boundary regularity of the Dirichlet problem. See Proposition 5.1.)

\subsection{Applications to Mathematical Finance}

The operators $(-\triangle)^{s}$ arise in stochastic theory as the operators associated with symmetric $\alpha$-stable Levy processes. Suppose we have such a Levy process $X_{t}$ such that $X_{0}=x$ for some point $x$ in $\mathbb{R}^{n}$. We consider the optimal stopping time $\tau$ to maximize the function

$$
u(x)=\sup _{\tau} E\left[\varphi\left(X_{\tau}\right): \tau<+\infty\right] .
$$

Then the function $u$ turns out to be the solution of our obstacle problem

- $u \geq \varphi$ in $\mathbb{R}^{n}$,

- $(-\triangle)^{s} u \geq 0$ in $\mathbb{R}^{n}$,

- $(-\triangle)^{s} u(x)=0$ for those $x$ such that $u(x)>\varphi(x)$,

- $\lim _{|x| \rightarrow+\infty} u(x)=0$.

If, on the other hand, we consider the problem

$$
u(x)=\sup _{\tau} E\left[e^{-\lambda \tau} \varphi\left(X_{\tau}\right)\right],
$$

then the function $u$ turns out to be the solution of the following obstacle problem:

- $u \geq \varphi$ in $\mathbb{R}^{n}$,

- $\lambda u+(-\triangle)^{s} u \geq 0$ in $\mathbb{R}^{n}$

- $\lambda u+(-\triangle)^{s} u(x)=0$ for those $x$ such that $u(x)>\varphi(x)$,

- $\lim _{|x| \rightarrow+\infty} u(x)=0$.

A problem like this arises in financial mathematics as a pricing model for American options. These models have become of increasing interest in the last few years. The function $u$ represents the rational price of a perpetual American option where the assets prices are modeled by a Levy process $X_{t}$ and the payoff function is $\varphi$. For nonperpetual options, a parabolic version of this problem is considered. A very readable explanation of these models can be found in the book by Cont and Tankov [6] (see also [10] and [11]). Usually the models are in one dimension, and 
although general payoff functions are considered, the case when $\varphi=\left(K-e^{x}\right)^{+}$ (the American put) is of special interest.

There is not much work done regarding regularity. In [2], S. Boyarchenko and $\mathrm{S}$. Levendorskiı̌ studied for which classes of Levy processes this problem has $C^{1}$ solutions (smooth pasting). They considered a very general family of (one-dimensional) Levy processes, and a class of payoff functions that assures that the contact set is a half-line.

When we consider jump processes whose corresponding integrodifferential operators have a kernel that coincides with $1 /|y|^{n+2 s}$ around the origin, then the solutions of the corresponding obstacle problem also satisfy an obstacle problem for the operator $(-\triangle)^{s}$ with a right-hand side. In many cases, we can assure enough regularity for that right-hand side, and the results of this work hold for those integrodifferential operators, too.

\section{Preliminary Properties of the Fractional Laplace Operator}

In this section, we provide some elementary properties of the operators $(-\triangle)^{\sigma}$ that we will need. The usual reference for these operators is Landkof's book [9]. We will show how $(-\triangle)^{\sigma}$ interacts with $C^{\alpha}$-norms, and characterize its supersolutions.

\subsection{Definitions and Properties}

Throughout this section $\mathcal{S}$ stands for the Schwartz space of rapidly decreasing $C^{\infty}$ functions in $\mathbb{R}^{n}$. Its dual, written as $\mathcal{S}^{\prime}$, is the space of tempered distributions in $\mathbb{R}^{n}$.

The following classical theorem about distributions is going to be used:

THEOREM 2.1 Suppose that a distribution $f$ is such that for any nonnegative test function $g,\langle f, g\rangle \geq 0$. Then $f$ is a nonnegative Radon measure in $\mathbb{R}^{n}$.

Two distributions $f$ and $g$ in $\mathbb{R}^{n}$ are said to coincide in an open set $\Omega$ if for every test function $\phi$ supported inside $\Omega$

$$
\langle f, \phi\rangle=\langle g, \phi\rangle .
$$

We recall the definition of $(-\triangle)^{\sigma}$ as a pseudodifferential operator.

Definition 2.2 Given $\sigma \in(-n / 2,1]$ and $f \in \mathcal{S}$, we define $(-\triangle)^{\sigma} f$ as

$$
\widehat{(-\triangle)^{\sigma}} f(\xi)=|\xi|^{2 \sigma} \widehat{f}(\xi) \text {. }
$$

Notice that $(-\triangle)^{\sigma} f \notin \mathcal{S}$ since $\mid \xi^{2 \sigma}$ introduces a singularity at the origin in its Fourier transform. That singularity is going to translate in a lack of rapid decay for $(-\triangle)^{\sigma} f$. However, $(-\triangle)^{\sigma} f$ is still $C^{\infty}$.

If $\sigma \leq-n / 2$, then $|\xi|^{2 \sigma}$ is not a tempered distribution, so we cannot allow that case. Technically we could define the case $\sigma>1$ this way, but we are not interested 
in this right now. Clearly, $(-\triangle)^{1}=-\triangle,(-\triangle)^{0}=\operatorname{Id}$ and $(-\triangle)^{\sigma_{1}} \circ(-\triangle)^{\sigma_{2}}=$ $(-\triangle)^{\sigma_{1}+\sigma_{2}}$.

We can also compute the same operator using a singular integral. When $f \in \mathcal{S}$ and $\sigma \in(0,1)$, we can compute $(-\triangle)^{\sigma} f$ as

$$
(-\triangle)^{\sigma} f(x)=c_{n, \sigma} \mathrm{PV} \int_{\mathbb{R}^{n}} \frac{f(x)-f(y)}{|x-y|^{n+2 \sigma}} \mathrm{d} y .
$$

The operator $(-\triangle)^{-\sigma}$ (for $\sigma>0$ ) can also be computed with an integral when $n>2 \sigma$ by

$$
(-\triangle)^{-\sigma} f(x)=c_{n,-\sigma} \int_{\mathbb{R}^{n}} \frac{f(y)}{|x-y|^{n-2 \sigma}} \mathrm{d} y .
$$

We refer to [9] for a detailed proof of the equivalence between (2.1) and (2.2) or (2.3). From (2.3), we see that $F(x)=c_{n,-\sigma} /|x|^{n-2 \sigma}$ is the fundamental solution of $(-\triangle)^{\sigma}$; i.e., $(-\triangle)^{\sigma} F=\delta_{0}$ when $n>2 \sigma$. This function is generally known as the Riesz kernel. From the definition of $(-\triangle)^{\sigma}$ in $\mathcal{S}$, we can extend it by duality in a large class of tempered distributions.

DEFINITION 2.3 Let $\overline{\mathcal{S}}_{\sigma}$ be the space of $C^{\infty}$-functions $f$ such that, for every $k \geq 0$, $\left(1+|x|^{n+2 \sigma}\right) f^{(k)}(x)$ is bounded. We consider the topology in $\overline{\mathcal{S}}_{\sigma}$ given by the family of seminorms

$$
[f]_{k}=\sup \left(1+|x|^{n+2 \sigma}\right) f^{(k)}(x) .
$$

We take $\overline{\mathcal{S}}_{\sigma}^{\prime}$ to be the dual of $\overline{\mathcal{S}}_{\sigma}$.

It is very simple to check that $(-\triangle)^{\sigma} f \in \overline{\mathcal{S}}_{\sigma}$ when $f \in \mathcal{S}$.

The symmetry of the operator $(-\triangle)^{\sigma}$ allows us to extend its definition to the space $\overline{\mathcal{S}}_{\sigma}^{\prime}$ by duality; i.e., if $u \in \overline{\mathcal{S}}_{\sigma}^{\prime}$,

$$
\left\langle(-\triangle)^{\sigma} u, f\right\rangle=\left\langle u,(-\triangle)^{\sigma} f\right\rangle
$$

This definition coincides with the previous ones in the case where $u \in \mathcal{S}$ and $(-\triangle)^{\sigma}$ is a continuous operator from $\overline{\mathcal{S}}_{\sigma}^{\prime}$ to $\mathcal{S}^{\prime}$.

We are rarely going to use these operators in such general spaces. But it is convenient to have in mind how far they can be extended. In general, we will be applying these operators to functions in $L_{\text {loc }}^{1}$. The natural space that we are going to use is a weighted $L^{1}$-space:

$$
L_{\sigma}:=L_{\mathrm{loc}}^{1} \cap \overline{\mathcal{S}}_{\sigma}^{\prime}=\left\{u: \mathbb{R}^{n} \rightarrow \mathbb{R} \text { such that } \int_{\mathbb{R}^{n}} \frac{|u(x)|}{1+|x|^{n+2 \sigma}} \mathrm{d} x<+\infty\right\} .
$$

The norm in $L_{\sigma}$ is naturally given by

$$
\|u\|_{L_{\sigma}}=\int_{\mathbb{R}^{n}} \frac{|u(x)|}{1+|x|^{n+2 \sigma}} \mathrm{d} x .
$$


In special cases, our formulas with the Fourier transform or the singular integrals are enough to compute the value. The following technical property exemplifies this situation:

PROpOSITION 2.4 Let $f$ be a function in $L_{\sigma}$ that is $C^{2 \sigma+\varepsilon}$ (or $C^{1,2 \sigma+\varepsilon-1}$ if $\sigma>\frac{1}{2}$ ) for some $\varepsilon>0$ in an open set $\Omega$; then for $\sigma \in(0,1),(-\triangle)^{\sigma} f$ is a continuous function in $\Omega$ and its values are given by the integral of (2.2).

PROOF: Let us take an arbitrary open set $\Omega_{0}$ compactly contained in $\Omega$. There exists a sequence $f_{k} \in S$ uniformly bounded in $C^{\sigma+\varepsilon}(\Omega)$ (or $C^{1, \sigma+\varepsilon-1}$ ), converging uniformly to $f$ in $\Omega_{0}$ and also converging to $f$ in the norm of $L_{\sigma}$. By the uniform bound on the $C^{\sigma+\varepsilon}$-norm of $f_{k}$ in $\Omega_{0}$, it can be shown that the integrals converge uniformly in $\Omega_{0}$,

$$
(-\triangle)^{\sigma} f_{k}(x)=\int_{\mathbb{R}^{n}} \frac{f_{k}(x)-f_{k}(y)}{|x-y|^{n+2 \sigma}} \mathrm{d} y \rightarrow \int_{\mathbb{R}^{n}} \frac{f(x)-f(y)}{|x-y|^{n+2 \sigma}} \mathrm{d} y .
$$

But $(-\triangle)^{\sigma} f_{k} \rightarrow(-\triangle)^{\sigma} f$ in the topology of $\mathcal{S}^{\prime}$. That implies that $(-\triangle)^{\sigma} f$ must coincide with the integral in $\Omega_{0}$ by the uniqueness of the limits. Since $\Omega_{0}$ is arbitrary, this happens for any $x \in \Omega$.

The following propositions explain how the operators $(-\triangle)^{\sigma}$ interact with $C^{\alpha}$ norms:

Proposition 2.5 Let $u \in C^{0, \alpha}\left(\mathbb{R}^{n}\right)$ for $\alpha \in(0,1]$ and $\alpha>2 \sigma>0$; then $(-\triangle)^{\sigma} u \in C^{0, \alpha-2 \sigma}$ and

$$
\left[(-\triangle)^{\sigma} u\right]_{C^{0, \alpha-2 \sigma}} \leq C[u]_{C^{0, \alpha}}
$$

where $C$ depends only on $\alpha, \sigma$, and $n$.

PROOF: Let us estimate the difference $\left|(-\triangle)^{\sigma} u\left(x_{1}\right)-(-\triangle)^{\sigma}\left(x_{2}\right)\right|$ for $x_{1}, x_{2} \in$ $\mathbb{R}^{n}$ :

$$
\begin{aligned}
& \left|(-\triangle)^{\sigma} u\left(x_{1}\right)-(-\triangle)^{\sigma} u\left(x_{2}\right)\right| \\
& \quad=C_{n, \sigma}\left|\int_{\mathbb{R}^{n}} \frac{u\left(x_{1}\right)-u\left(x_{1}+y\right)-u\left(x_{2}\right)+u\left(x_{2}+y\right)}{|y|^{n+2 \sigma}} \mathrm{d} y\right| \\
& \quad \leq \mathrm{I}_{1}+\mathrm{I}_{2},
\end{aligned}
$$

where

$$
\begin{aligned}
& \mathrm{I}_{1}=C_{n, \sigma}\left|\int_{B_{r}} \frac{u\left(x_{1}\right)-u\left(x_{1}+y\right)-u\left(x_{2}\right)+u\left(x_{2}+y\right)}{|y|^{n+2 \sigma}} \mathrm{d} y\right|, \\
& \mathrm{I}_{2}=C_{n, \sigma}\left|\int_{\mathbb{R}^{n} \backslash B_{r}} \frac{u\left(x_{1}\right)-u\left(x_{1}+y\right)-u\left(x_{2}\right)+u\left(x_{2}+y\right)}{|y|^{n+2 \sigma}} \mathrm{d} y\right| .
\end{aligned}
$$


For $\mathrm{I}_{1}$, we use that $\left|u\left(x_{i}\right)-u\left(x_{i}+y\right)\right| \leq[u]_{C^{0, \alpha}}|y|^{\alpha}$ for $i=1$, 2 . Therefore

$$
\begin{aligned}
\mathrm{I}_{1} & \leq C_{n, \sigma}\left|\int_{B_{r}} \frac{2[u]_{C^{0, \alpha}|y|^{\alpha}}}{|y|^{n+2 \sigma}}\right| \\
& \leq C[u]_{C^{0, \alpha}} r^{\alpha-2 \sigma} .
\end{aligned}
$$

For $\mathrm{I}_{2}$, we use that $\left|u\left(x_{1}+y\right)-u\left(x_{2}+y\right)\right| \leq[u]_{C^{0, \alpha}}\left|x_{1}-x_{2}\right|^{\alpha}$. Therefore

$$
\begin{aligned}
\mathrm{I}_{1} & \leq C_{n, \sigma}\left|\int_{\mathbb{R}^{n} \backslash B_{r}} \frac{2[u]_{C^{0, \alpha}}\left|x_{1}-x_{2}\right|^{\alpha}}{|y|^{n+2 \sigma}}\right| \\
& \leq C[u]_{C^{0, \alpha}} r^{-2 \sigma}\left|x_{1}-x_{2}\right|^{\alpha} .
\end{aligned}
$$

Picking $r=\left|x_{1}-x_{2}\right|$ and adding $\mathrm{I}_{1}$ and $\mathrm{I}_{2}$, we obtain

$$
\left|(-\triangle)^{\sigma} u\left(x_{1}\right)-(-\triangle)^{\sigma}\left(x_{2}\right)\right| \leq C[u]_{C^{0, \alpha}}\left|x_{1}-x_{2}\right|^{\alpha-2 \sigma} .
$$

Proposition 2.6 Let $u \in C^{1, \alpha}\left(\mathbb{R}^{n}\right)$ for $\alpha \in(0,1]$ and $\sigma>0$.

- If $\alpha>2 \sigma$, then $(-\triangle)^{\sigma} u \in C^{1, \alpha-2 \sigma}$ and

$$
\left[(-\triangle)^{\sigma} u\right]_{C^{1, \alpha-2 \sigma}} \leq C[u]_{C^{1, \alpha}}
$$

where $C$ depends only on $\alpha, \sigma$, and $n$.

- If $\alpha<2 \sigma$, then $(-\triangle)^{\sigma} u \in C^{0, \alpha-2 \sigma+1}$ and

$$
\left[(-\triangle)^{\sigma} u\right]_{C^{0, \alpha-2 \sigma+1}} \leq C[u]_{C^{1, \alpha}}
$$

where $C$ depends only on $\alpha, \sigma$, and $n$.

PROOF: The first part follows simply by Proposition 2.5 plus the fact that the operators $(-\triangle)^{\sigma}$ commute with differentiation.

For the second part, let us first assume that $\sigma<\frac{1}{2}$. We proceed as in the proof of Proposition 2.5 to get

$$
\left|(-\triangle)^{\sigma} u\left(x_{1}\right)-(-\triangle)^{\sigma}\left(x_{2}\right)\right| \leq \mathrm{I}_{1}+\mathrm{I}_{2}
$$

for the same $\mathrm{I}_{1}$ and $\mathrm{I}_{2}$ as before. But now to estimate $\mathrm{I}_{1}$ we use that since $u \in C^{1, \alpha}$,

$$
\begin{aligned}
& \left|u\left(x_{1}\right)-u\left(x_{1}+y\right)-u\left(x_{2}\right)+u\left(x_{2}+y\right)\right| \\
& \quad \leq\left|\left(\nabla u\left(x_{1}\right)-\nabla u\left(x_{2}\right)\right) \cdot y\right|+[u]_{C^{1, \alpha}}|y|^{1+\alpha} \\
& \quad \leq[u]_{C^{1, \alpha}}\left(|y|\left|x_{1}-x_{2}\right|^{\alpha}+|y|^{1+\alpha}\right) ;
\end{aligned}
$$

then $\mathrm{I}_{1} \leq C[u]_{C^{1, \alpha}}\left(r^{1-2 \sigma}\left|x_{1}-x_{2}\right|^{\alpha}+r^{1+\alpha-2 \sigma}\right)$.

In the case $\sigma \geq \frac{1}{2}$, we write $(-\triangle)^{\sigma}=(-\triangle)^{\sigma-1 / 2} \circ(-\triangle)^{1 / 2}$, and the result follows from the observation that $(-\triangle)^{1 / 2}=\sum_{i} R_{i} \partial_{i}$, where the $R_{i}$ are the Riesz transforms.

Iterating the last two propositions we get the following result: 
Proposition 2.7 Let $u \in C^{k, \alpha}$ and suppose that $k+\alpha-2 \sigma$ is not an integer. Then $(-\triangle)^{\sigma} u \in C^{l, \beta}$ where $l$ is the integer part of $k+\alpha-2 \sigma$ and $\beta=k+\alpha-2 \sigma-l$.

Proposition 2.8 Let $w=(-\triangle)^{\sigma} u$. Assume $w \in C^{0, \alpha}\left(\mathbb{R}^{n}\right)$ and $u \in L^{\infty}$ for $\alpha \in(0,1]$ and $\sigma>0$.

- If $\alpha+2 \sigma \leq 1$, then $u \in C^{0, \alpha+2 \sigma}\left(\mathbb{R}^{n}\right)$. Moreover,

$$
\|u\|_{C^{0, \alpha+2 \sigma}\left(\mathbb{R}^{n}\right)} \leq C\left(\|u\|_{L^{\infty}}+\|w\|_{C^{0, \alpha}}\right)
$$

for a constant $C$ depending only on $n, \alpha$, and $\sigma$.

- If $\alpha+2 \sigma>1$, then $u \in C^{1, \alpha+2 \sigma-1}\left(\mathbb{R}^{n}\right)$. Moreover,

$$
\|u\|_{C^{1, \alpha+2 \sigma-1}\left(\mathbb{R}^{n}\right)} \leq C\left(\|u\|_{L^{\infty}}+\|w\|_{C^{0, \alpha}}\right)
$$

for a constant $C$ depending only on $n, \alpha$, and $\sigma$.

PROOF: We will show that $u$ has the corresponding regularity in a neighborhood of the origin. The same argument works for a neighborhood of every point; so we get, respectively, that $u \in C^{0, \alpha+2 \sigma}\left(\mathbb{R}^{n}\right)$ or $u \in C^{1, \alpha+2 \sigma-1}\left(\mathbb{R}^{n}\right)$.

Let $\eta$ be a smooth cutoff function such that $\eta(x) \in[0,1]$ for every $x \in \mathbb{R}^{n}$, supp $\eta \subset B_{2}$, and $\eta(x)=1$ for every $x \in B_{1}$. Let

$$
u_{0}(x):=c_{n,-\sigma} \int_{\mathbb{R}^{n}} \frac{\eta(y) w(y)}{|x-y|^{n-2 \sigma}} \mathrm{d} y=(-\triangle)^{-\sigma} \eta w(x) .
$$

Then $(-\triangle)^{\sigma} u_{0}=w=(-\triangle)^{\sigma} u$ in $B_{1}$, and therefore $u-u_{0}$ is smooth in $B_{1 / 2}$. Moreover, its $C^{0, \alpha+2 \sigma}$ - or $C^{1, \alpha+2 \sigma-1}$-norm can be estimated from the $L^{\infty}$-norm of $u_{0}-u$, which can be estimated from the $L^{\infty}$-norms of $u$ and $w$.

So, we are only left to show that $u_{0} \in C^{0, \alpha+2 \sigma}\left(B_{1 / 2}\right)$. Assume $\alpha<1$; then we write $u_{0}=(\triangle)^{-\sigma} \eta w=(-\triangle)^{1-\sigma} \circ(-\triangle)^{-1} \eta w$, and from the $C^{2, \alpha}$-estimates for the Poisson equation (see [8]) we know that $(-\triangle)^{-1} \eta w \in C^{2, \alpha}$ and its norm depends only on $\|w\|_{C_{0, \alpha}}$. Now we apply Proposition 2.7 and we conclude the proof. On the other hand, if $\alpha=1$, then $\alpha>1-2 \sigma$, and we write $u_{0}=(-\triangle)^{-1} \circ$ $(-\triangle)^{1-\sigma} \eta w$; the result follows from Proposition 2.7 and the $C^{2, \alpha}$-estimates for the Poisson equation.

Proposition 2.9 Let $w=(-\triangle)^{\sigma} u$. Assume $w \in L^{\infty}\left(\mathbb{R}^{n}\right)$ and $u \in L^{\infty}$ for $\sigma>0$.

- If $2 \sigma \leq 1$, then $u \in C^{0, \alpha}\left(\mathbb{R}^{n}\right)$ for any $\alpha<2 \sigma$. Moreover,

$$
\|u\|_{C^{0, \alpha}\left(\mathbb{R}^{n}\right)} \leq C\left(\|u\|_{L^{\infty}}+\|w\|_{L^{\infty}}\right)
$$

for a constant $C$ depending only on $n, \alpha$, and $\sigma$.

- If $2 \sigma>1$, then $u \in C^{1, \alpha}\left(\mathbb{R}^{n}\right)$ for any $\alpha<2 \sigma-1$. Moreover,

$$
\|u\|_{C^{1, \alpha}\left(\mathbb{R}^{n}\right)} \leq C\left(\|u\|_{L^{\infty}}+\|w\|_{L^{\infty}}\right)
$$

for a constant $C$ depending only on $n, \alpha$, and $\sigma$. 
PROOF: The proof is identical to the one for Proposition 2.8 with the difference that we have to use $C^{1, \alpha}$-estimates for the Poisson equation with the right-hand side in $L^{\infty}$ instead of $C^{2, \alpha}$-estimates.

\subsection{Supersolutions and Comparison}

We want the least restrictive possible definition of supersolutions for the equation

$$
(-\triangle)^{\sigma} u \geq 0
$$

so that we can prove general theorems of comparison. We want to be able to apply maximum principles to nonsmooth functions for which the integral representation (2.2) of $(-\triangle)^{\sigma}$ does not apply. We also want to be able to check (2.4) in an open domain $\Omega$ that is not the whole space $\mathbb{R}^{n}$. We will obtain characterizations of supersolutions similar to the mean value for superharmonic functions that we will use later in the paper.

When we are interested in the whole space, (2.4) means of course that $(-\triangle)^{\sigma} u$ is a nonnegative measure.

DEFINITION 2.10 We say that $u \in \overline{\mathcal{S}}_{\sigma}^{\prime}$ satisfies $(-\triangle)^{\sigma} u \geq 0$ in an open set $\Omega$ if for every nonnegative test function $\phi$ whose support is inside $\Omega,\left\langle u,(-\triangle)^{\sigma} \phi\right\rangle \geq 0$.

The definition says that $(-\triangle)^{\sigma} u$ coincides with a nonnegative Radon measure in $\Omega$. This is good for a definition but it is awkward to deal with. We would like to have a property like the definition of superharmonic functions comparing the value at a point with the means in small balls centered there. We will restrict our study to functions $u \in L_{\sigma}$. We are going to use some special test functions.

Let $\Phi(x)=C /|x|^{n-2 \sigma}$ be the fundamental solution of $(-\triangle)^{\sigma}$. Let us stick a paraboloid from below to cut out the singularity at $x=0$ to obtain a $C^{1,1}$-function $\Gamma(x)$ that coincides with $\Phi(x)$ when $x$ is outside the ball of radius 1 centered at the origin (see Figure 2.1).

Given $\lambda>1$, consider $\Gamma_{\lambda}=\Gamma(x / \lambda) / \lambda^{n-2 \sigma}$. The function $\Gamma_{\lambda} \in C^{1,1}$ coincides with $\Phi$ outside of the ball of radius $\lambda$ centered at the origin, and it is a paraboloid inside that ball. Besides, $\Gamma_{\lambda_{1}} \geq \Gamma_{\lambda_{2}}$ if $\lambda_{1} \leq \lambda_{2}$.

We need the next proposition in order to use $(-\triangle)^{\sigma} \Gamma_{\lambda}$ as an approximation of the identity.

PROPOSITION $2.11(-\triangle)^{\sigma} \Gamma$ is a positive continuous function in $L^{1}$. Thus,

$$
(-\triangle)^{\sigma} \Gamma \geq 0 .
$$

In addition, $\int_{\mathbb{R}^{n}}(-\triangle)^{\sigma} \Gamma(x) \mathrm{d} x=1$.

PROOF: Since $\Gamma$ is $C^{1,1}$, we can use the integral representation (2.2) to compute $(-\triangle)^{\sigma} \Gamma$. If $x_{0} \notin B_{1}$, then $\Gamma\left(x_{0}\right)=\Phi\left(x_{0}\right)$; for every other $x, \Gamma(x) \leq \Phi(x)$. Then

$$
(-\triangle)^{\sigma} \Gamma\left(x_{0}\right)=\int_{\mathbb{R}^{n}} \frac{\Gamma\left(x_{0}\right)-\Gamma(y)}{\left|x_{0}-y\right|^{n+2 \sigma}} \mathrm{d} y
$$




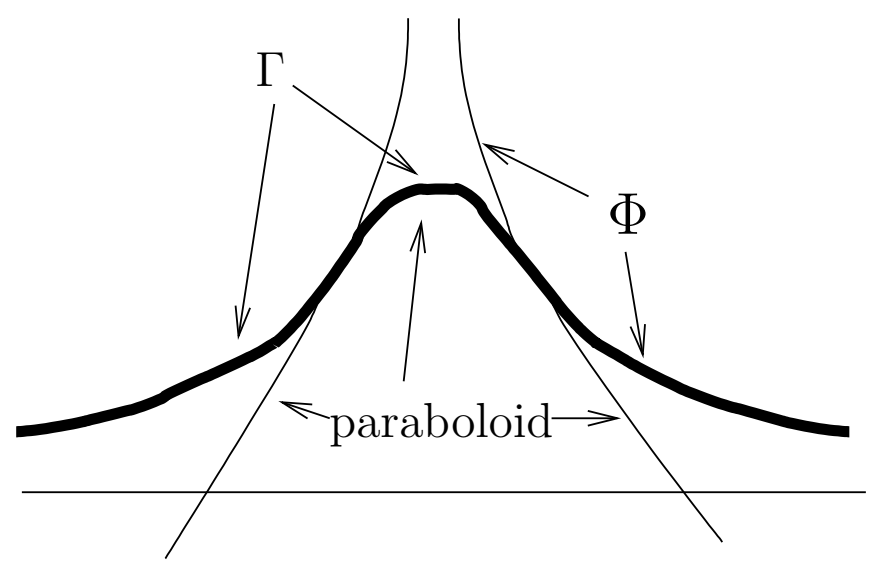

FIGURE 2.1. The function $\Gamma$.

$$
>\int_{\mathbb{R}^{n}} \frac{\Phi\left(x_{0}\right)-\Phi(y)}{\left|x_{0}-y\right|^{n+2 \sigma}} \mathrm{d} y=0
$$

since $\Phi$ is the fundamental solution.

If $x_{0} \in B_{1}-\{0\}$, there exist an $x_{1}$ and a positive $\delta$ such that $\Phi\left(x-x_{1}\right)+\delta$ touches $\Gamma$ from above at the point $x_{0}$. Now we use the singular integral representation

$$
\begin{aligned}
(-\triangle)^{\sigma} \Gamma\left(x_{0}\right) & =\int_{\mathbb{R}^{n}} \frac{\Gamma\left(x_{0}\right)-\Gamma(y)}{\left|x_{0}-y\right|^{n+2 \sigma}} \mathrm{d} y \\
& >\int_{\mathbb{R}^{n}} \frac{\Phi\left(x_{0}-x_{1}\right)+\delta-\Phi\left(y-x_{1}\right)-\delta}{\left|x_{0}-y\right|^{n+2 \sigma}} \mathrm{d} y=0
\end{aligned}
$$

since $(-\triangle)^{\sigma}\left(\Phi\left(x_{0}-x_{1}\right)+\delta\right)=0$.

If $x_{0}=0$, then $\Gamma$ attains its maximum at $x_{0}$,

$$
(-\triangle)^{\sigma} \Gamma\left(x_{0}\right)=\int_{\mathbb{R}^{n}} \frac{\Gamma\left(x_{0}\right)-\Gamma(y)}{\left|x_{0}-y\right|^{n+2 \sigma}} \mathrm{d} y>0,
$$

because we are integrating a positive function.

To show that $\int_{\mathbb{R}^{n}}(-\triangle)^{\sigma} \Gamma(x) \mathrm{d} x=1$, we consider a smooth cutoff function $\eta$ such that $\eta(x) \leq 1$ for every $x \in \mathbb{R}^{n}, \eta(x)=1$ for every $x \in B_{1}$ and $\operatorname{supp} \eta \subset B_{2}$. Let $\eta_{R}(x)=\eta(x / R)$; then we have

$$
\begin{aligned}
\int_{\mathbb{R}^{n}}(-\triangle)^{\sigma} \Gamma(x) \mathrm{d} x-1 & =\lim _{R \rightarrow \infty}\left\langle(-\triangle)^{\sigma} \Gamma-(-\triangle)^{\sigma} \Phi, \eta_{R}\right\rangle \\
& =\lim _{R \rightarrow \infty}\left\langle\Gamma-\Phi,(-\triangle)^{\sigma} \eta_{R}\right\rangle=0
\end{aligned}
$$


since clearly $(-\triangle)^{\sigma} \eta_{R}$ goes to 0 uniformly on compact sets, and $\Gamma-\Phi$ is an $L^{1}$-function with compact support.

Let $\gamma_{\lambda}=(-\triangle)^{\sigma} \Gamma_{\lambda}$.

Proposition 2.12 For any $\lambda$, the function $\gamma_{\lambda}(x)$ decays like $1 /|x|^{n+2 \sigma}$ when $x \rightarrow$ $\infty$.

PROOF: For $|x|$ large, $\Gamma_{\lambda}(x)=\Phi(x)$ and

$$
\begin{aligned}
\gamma_{\lambda}(x) & =\int \frac{\Gamma_{\lambda}(x)-\Gamma_{\lambda}(y)}{|x-y|^{n+2 \sigma}} \mathrm{d} y \\
& =\int \frac{\Phi(x)-\Phi(y)}{|x-y|^{n+2 \sigma}} \mathrm{d} y+\int \frac{\Phi(y)-\Gamma_{\lambda}(y)}{|x-y|^{n+2 \sigma}} \mathrm{d} y \\
& =\int \frac{\Phi(y)-\Gamma_{\lambda}(y)}{|x-y|^{n+2 \sigma}} \mathrm{d} y \\
& \cong \frac{1}{|x|^{n+2 \sigma}}
\end{aligned}
$$

since $\Phi(y)-\Gamma_{\lambda}(y)$ is a compactly supported function in $L^{1}$.

Proposition 2.13 The family $\gamma_{\lambda}$ is an approximation of the identity as $\lambda \rightarrow 0$. In the sense that

$$
u * \gamma_{\lambda}(x)=\int_{\mathbb{R}^{n}} u(y) \gamma_{\lambda}(x-y) \mathrm{d} y \rightarrow u(x) \quad \text { a.e. as } \lambda \rightarrow 0 .
$$

PROOF: First of all, notice that $u(y) \gamma_{\lambda}(x-y)$ is integrable for every $x$ since $u \in L_{\sigma}$ and $\gamma_{\lambda}$ decays as $1 /\left(1+|x|^{n+2 \sigma}\right)$ by Proposition 2.12 .

We have to check the rescaling properties of $\gamma_{\lambda}=(-\triangle)^{\sigma} \Gamma_{\lambda}$ :

$$
\begin{aligned}
\gamma_{\lambda}(x) & =(-\triangle)^{\sigma} \Gamma_{\lambda}(x)=(-\triangle)^{\sigma}\left(\frac{1}{\lambda^{n-2 \sigma}} \Gamma\left(\frac{x}{\lambda}\right)\right) \\
& =\frac{1}{\lambda^{n}}\left((-\triangle)^{\sigma} \Gamma\right)\left(\frac{x}{\lambda}\right)=\frac{1}{\lambda^{n}} \gamma_{1}\left(\frac{x}{\lambda}\right) .
\end{aligned}
$$

Since $\gamma_{1}$ is nonnegative and $\int \gamma_{1} \mathrm{~d} x=1$, we conclude the proof.

PROPOSITION 2.14 If $(-\triangle)^{\sigma} u$ is continuous at a point $x \in \mathbb{R}^{n}$, then

$$
(-\triangle)^{\sigma} u(x)=\lim _{\lambda \rightarrow 0} \frac{C}{\lambda^{n}}\left(u(x)-u * \gamma_{\lambda}(x)\right),
$$

where the constant $C$ depends only on $\sigma$ and $n$.

PROOF: Since $(-\triangle)^{\sigma} u$ is continuous at the point $x$, it is bounded in a neighborhood of $x$, and for any function $g \in L^{1}\left(\mathbb{R}^{n}\right)$ with compact support

$$
\lim _{\lambda \rightarrow 0} \int_{\mathbb{R}^{n}}(-\triangle)^{\sigma} u(x-y) \frac{1}{\lambda^{n}} g\left(\frac{y}{\lambda}\right) \mathrm{d} y=(-\triangle)^{\sigma} u(x) \cdot \int_{\mathbb{R}^{n}} g \mathrm{~d} x .
$$


Let $g(y)=\Phi(y)-\Gamma(y)$; then

$$
\begin{aligned}
\frac{1}{\lambda^{n}} g\left(\frac{y}{\lambda}\right) & =\frac{1}{\lambda^{n}} \Phi\left(\frac{y}{\lambda}\right)-\frac{1}{\lambda^{n}} \Gamma\left(\frac{y}{\lambda}\right) \\
& =\frac{1}{\lambda^{2 \sigma}}\left(\Phi(y)-\Gamma_{\lambda}(y)\right) .
\end{aligned}
$$

Thus, substituting in (2.5),

$$
\begin{aligned}
(-\triangle)^{\sigma} u(x) & =C \lim _{\lambda \rightarrow 0} \frac{1}{\lambda^{2 \sigma}} \int_{\mathbb{R}^{n}}(-\triangle)^{\sigma} u(x-y)\left(\Phi(y)-\Gamma_{\lambda}(y)\right) \mathrm{d} y \\
& =\lim _{\lambda \rightarrow 0} \frac{C}{\lambda^{2 \sigma}}\left(u(x)-u * \gamma_{\lambda}(x)\right) .
\end{aligned}
$$

PROPOSITION 2.15 Given a function $u \in L_{\sigma},(-\triangle)^{\sigma} u \geq 0$ in an open set $\Omega$ if and only if $u$ is lower-semicontinuous in $\Omega$ and

$$
u\left(x_{0}\right) \geq \int_{\mathbb{R}^{n}} u(x) \gamma_{\lambda}\left(x-x_{0}\right) \mathrm{d} x
$$

for any $x_{0}$ in $\Omega$ and $\lambda \leq \operatorname{dist}\left(x_{0}, \partial \Omega\right)$.

PROOF: We would like to test $(-\triangle)^{\sigma} u$ against $\Phi-\Gamma_{\lambda}$ and "integrate by parts." Unfortunately, this may not be a valid test function. The next few paragraphs overcome this technical difficulty.

Let us consider a function $u$ such that

$$
\int_{\mathbb{R}^{n}} \frac{|u(x)|}{1+|x|^{n+2 \sigma}} \mathrm{d} x<+\infty .
$$

If $r>\lambda_{1}>\lambda_{2}, \Gamma_{\lambda_{2}}-\Gamma_{\lambda_{1}}$ is a nonnegative $C^{1,1}$-function supported in $B_{r}$. If $(-\triangle)^{\sigma} u \geq 0$ in $B_{r}\left(x_{0}\right)$, then

$$
\left\langle(-\triangle)^{\sigma} u, \Gamma_{\lambda_{2}}\left(x-x_{0}\right)-\Gamma_{\lambda_{1}}\left(x-x_{0}\right)\right\rangle \geq 0 .
$$

Using the self-adjointness of $(-\triangle)^{\sigma}$, we have

$$
\left\langle u,(-\triangle)^{\sigma} \Gamma_{\lambda_{2}}\left(x-x_{0}\right)-(-\triangle)^{\sigma} \Gamma_{\lambda_{1}}\left(x-x_{0}\right)\right\rangle \geq 0 .
$$

Therefore

$$
\begin{aligned}
\left\langle u, \gamma_{\lambda_{2}}\left(x-x_{0}\right)\right\rangle & \geq\left\langle u, \gamma_{\lambda_{1}}\left(x-x_{0}\right)\right\rangle, \\
u * \gamma_{\lambda_{2}}\left(x_{0}\right) & \geq u * \gamma_{\lambda_{1}}\left(x_{0}\right) .
\end{aligned}
$$

Let $\Omega_{0} \Subset \Omega$ and $(-\triangle)^{\sigma} u \geq 0$ in $\Omega$. Let $r=\operatorname{dist}\left(\Omega_{0}, \partial \Omega\right)$. Then if $r>\lambda_{1}>$ $\lambda_{2}>0$,

$$
u * \gamma_{\lambda_{2}} \geq u * \gamma_{\lambda_{1}} \text { in } \Omega_{0} .
$$

But $\gamma_{\lambda}$ is an approximate identity, $u * \gamma_{\lambda} \rightarrow u$ a.e. in $\Omega_{0}$ as $\lambda \rightarrow 0$. 
For each $\lambda, u * \gamma_{\lambda}$ is continuous. So $u$ is the limit of an increasing sequence of continuous functions. That means that (possibly modifying $u$ in a set of measure zero), $u$ is lower-semicontinuous.

Taking $\lambda_{2} \rightarrow 0$ in (2.6), we obtain the important property of supersolutions of the operator $(-\triangle)^{\sigma}$ that replaces the mean value property of the classical Laplace operator:

$$
u * \gamma_{\lambda}\left(x_{0}\right) \leq u\left(x_{0}\right) \quad \text { for every } x_{0} \in \Omega \text { and } \lambda \text { small enough. }
$$

The "if" part is already proved when $\lambda<\operatorname{dist}\left(x_{0}, \partial \Omega\right.$ ) (notice that $\gamma$ is symmetric). The case $\lambda=\operatorname{dist}\left(x_{0}, \partial \Omega\right)$ follows by passage to the limit. The "only if" part follows easily.

COROLLARY 2.16 There is a constant $C$ such that for every $x \in \Omega$,

$$
u(x) \geq u * \gamma_{\lambda}(x)-C \lambda^{2 s} \text { for every } \lambda<\operatorname{dist}(x, \partial \Omega)
$$

if and only if $(-\triangle)^{\sigma} u \geq-C$ in $\Omega$ (in the sense that $(-\triangle)^{\sigma} u+C$ is a nonnegative Radon measure).

PROOF: We can assume that $\Omega$ is bounded (since $f \geq-C$ locally in $\Omega$ is the same as $f \geq-C$ in the whole $\Omega$ for any distribution $f$ ). Let $v=C \Phi * \chi_{\Omega}$ so that $(-\triangle)^{\sigma} v=C \chi_{\Omega}$. By Proposition 2.14, for any $x \in \Omega$,

$$
C=\lim _{\lambda \rightarrow 0} \frac{1}{\lambda^{2 \sigma}}\left(v(x)-v * \gamma_{\lambda}(x)\right) .
$$

But actually we can see that since $(-\triangle)^{\sigma} v$ is constant in $\Omega$ and $\Phi-\Gamma_{\lambda}$ is supported in $B_{\lambda}$, then

$$
C=\frac{1}{\lambda^{2 \sigma}}\left(v(x)-v * \gamma_{\lambda}(x)\right)
$$

for $\lambda<\operatorname{dist}(x, \partial \Omega)$.

Now we consider $u+v$; then $u+v(x) \geq(u+v) * \gamma_{\lambda}(x)$ is equivalent to (2.8), which means that $(-\triangle)^{\sigma}(u+v) \geq 0$ in $\Omega$. Thus (2.8) holds if and only if $(-\triangle)^{\sigma}(u+v) \geq 0$, i.e., $(-\triangle)^{\sigma} u \geq-C$ in $\Omega$.

With Proposition 2.15 in mind, we can prove the basic properties of supersolutions for the operator $(-\triangle)^{\sigma}$ without requiring the singular integrals to be welldefined. We now show a maximum principle.

Proposition 2.17 Let $\Omega \Subset \mathbb{R}^{n}$ be an open set, and let $u$ be a lower-semicontinuous function in $\bar{\Omega}$ such that $(-\triangle)^{\sigma} u \geq 0$ in $\Omega$ and $u \geq 0$ in $\mathbb{R}^{n} \backslash \Omega$. Then $u \geq 0$ in $\mathbb{R}^{n}$. Moreover, if $u(x)=0$ for one point $x$ inside $\Omega$, then $u \equiv 0$ in all $\mathbb{R}^{n}$.

Proof: We need semicontinuity in $\bar{\Omega}$ because we cannot assure that any superharmonic function will be semicontinuous up to the boundary of the domain.

If $u$ takes negative values in $\mathbb{R}^{n}$, then they must all lie inside $\Omega$. Since $u$ is lower-semicontinuous, it attains its minimum in $\bar{\Omega}$ (which is a compact set in $\mathbb{R}^{n}$ ). 
Suppose that the minimum is negative and is attained at a point $x_{0} \in \Omega$. Then by Proposition 2.15 , there is a $\lambda$ such that

$$
u\left(x_{0}\right) \geq \int_{\mathbb{R}^{n}} u(x) \gamma_{\lambda}\left(x-x_{0}\right) \mathrm{d} x .
$$

But $\gamma_{\lambda}$ is strictly positive and has integral 1 , so

$$
0 \geq \int_{\mathbb{R}^{n}}\left(u(x)-u\left(x_{0}\right)\right) \gamma_{\lambda}\left(x-x_{0}\right) \mathrm{d} x .
$$

That is impossible because since $u\left(x_{0}\right)<0$, the right-hand side is strictly positive.

Now, if $u\left(x_{0}\right)=0$, we get

$$
0 \geq \int_{\mathbb{R}^{n}} u(x) \gamma_{\lambda}\left(x-x_{0}\right) \mathrm{d} x .
$$

But because $u(x)$ is nonnegative,

$$
0 \leq \int_{\mathbb{R}^{n}} u(x) \gamma_{\lambda}\left(x-x_{0}\right) \mathrm{d} x .
$$

Therefore

$$
0=\int_{\mathbb{R}^{n}} u(x) \gamma_{\lambda}\left(x-x_{0}\right) \mathrm{d} x .
$$

Since $\gamma_{\lambda}$ is strictly positive, we obtain $u \equiv 0$.

PROPOSITION 2.18 If $u_{1}, u_{2} \in L_{\sigma}$ are two supersolutions for the operator $(-\triangle)^{\sigma}$ in $\Omega$ (i.e., $(-\triangle)^{\sigma} u \geq 0$ and $(-\triangle)^{\sigma} v \geq 0$ in $\Omega$ ), then $u(x)=\min \left(u_{1}(x), u_{2}(x)\right)$ is too.

Proof: Given $x_{0} \in \Omega$, then $u\left(x_{0}\right)=u_{i}\left(x_{0}\right)$ for $i=1$ or $i=2$. By Proposition 2.15 for $\lambda$ small enough,

$$
u_{i}\left(x_{0}\right) \geq \int_{\mathbb{R}^{n}} u_{i}(x) \gamma_{\lambda}\left(x-x_{0}\right) \mathrm{d} x .
$$

But $u_{i}\left(x_{0}\right)=u\left(x_{0}\right)$ and $u(x) \leq u_{i}(x)$ for every other $x$. Then

$$
u\left(x_{0}\right) \geq \int_{\mathbb{R}^{n}} u_{i}(x) \gamma_{\lambda}\left(x-x_{0}\right) \mathrm{d} x \geq \int_{\mathbb{R}^{n}} u(x) \gamma_{\lambda}\left(x-x_{0}\right) \mathrm{d} x
$$

and $(-\triangle)^{\sigma} u \geq 0$.

For functions $u$ such that $(-\triangle)^{\sigma} u \leq 0$, a similar property holds:

Proposition 2.19 Given a function $u \in L_{\sigma},(-\triangle)^{\sigma} u \leq 0$ in an open set $\Omega$ if and only if $u$ is upper-semicontinuous in $\Omega$ and $u * \gamma_{\lambda}\left(x_{0}\right) \geq u\left(x_{0}\right)$ for any $x_{0}$ in $\Omega$ and $\lambda \leq \operatorname{dist}\left(x_{0}, \partial \Omega\right)$. 
We can also obtain the analogue of Corollary 2.16.

COROLLARY 2.20 There is a constant $C$ such that for all $\lambda \leq \operatorname{dist}\left(x_{0}, \partial \Omega\right)$ and $x \in \Omega, u(x) \leq u * \gamma_{\lambda}(x)+C \lambda^{2 s}$ if and only if $(-\triangle)^{\sigma} u \leq C$ in $\Omega$.

PROPOSITION 2.21 Let $\Omega \Subset \mathbb{R}^{n}$ be an open set, $(-\triangle)^{\sigma} u \geq 0$ and $(-\triangle)^{\sigma} v \leq 0$ in $\Omega$, such that $u \geq v$ in $\mathbb{R}^{n} \backslash \Omega$, and $u-v$ is lower-semicontinuous in $\bar{\Omega}$. Then $u \geq v$ in $\mathbb{R}^{n}$. Moreover, if $u(x)=v(x)$ for one point $x$ inside $\Omega$, then $u \equiv v$ in all $\mathbb{R}^{n}$.

ProOF: Apply Proposition 2.17 to $u-v$.

We have a similar property for functions $u$ such that $(-\triangle)^{\sigma} u=0$ in an open set $\Omega$.

Proposition 2.22 Given a function $u \in L_{\sigma},(-\triangle)^{\sigma} u=0$ in an open set $\Omega$ if and only if $u$ is continuous in $\Omega$ and $u * \gamma_{\lambda}\left(x_{0}\right)=u\left(x_{0}\right)$ for any $x_{0}$ in $\Omega$ and $\lambda \leq \operatorname{dist}\left(x_{0}, \partial \Omega\right)$.

From the above proposition, with a standard convolution argument we can get an iterative gain in regularity and prove that a function $u$ such that $(-\triangle)^{\sigma} u=0$ in an open set $\Omega$ is $C^{\infty}$ in that set. This is a well-known result not only for the fractional Laplacian but for any pseudodifferential operator.

Remark 2.23. We are not going to compute $\gamma_{\lambda}$ explicitly. The properties shown so far are enough for all our purposes. In [9], functions $u$ such that $(-\triangle)^{\sigma} u \geq 0$ are defined in a similar (and equivalent) way using some function in place of $\gamma_{\lambda}$ that is explicitly computed.

\section{Basic Properties of the Free Boundary Problem}

In this section we will construct a solution to our problem and show the first regularity results.

\subsection{Construction of the Solution}

We recall the statement of the problem that we are going to study.

Let $\varphi: \mathbb{R}^{n} \rightarrow \mathbb{R}$ be a continuous function with compact support ${ }^{1}$ that we will consider the obstacle. We look for a continuous function $u$ satisfying

- $u \geq \varphi$ in $\mathbb{R}^{n}$,

- $(-\triangle)^{s} u \geq 0$ in $\mathbb{R}^{n}$,

- $(-\triangle)^{s} u(x)=0$ for those $x$ such that $u(x)>\varphi(x)$,

- $\lim _{|x| \rightarrow+\infty} u(x)=0$.

\footnotetext{
${ }^{1}$ Rapid decay at infinity would suffice.
} 
We will prove that for any such $\varphi$, there is a solution $u$ to this problem. The proof fails when $n=1$ and $s>\frac{1}{2}$, because in that case it is impossible to have $(-\triangle)^{s} u \geq 0$ in $\mathbb{R}^{n}$ and at the same time for $u$ to vanish at infinity.

We will construct $u$ as the function that minimizes

$$
J(u):=\int_{\mathbb{R}^{n}} \int_{\mathbb{R}^{n}} \frac{|u(x)-u(y)|^{2}}{|x-y|^{n+2 s}} \mathrm{~d} x \mathrm{~d} y
$$

over all the functions $u \in \dot{H}^{s}$ that satisfy $\varphi \leq u$.

For any function $f \in \mathcal{S}$, the norm in $\dot{H}^{s}$ is given precisely by

$$
\|f\|_{\dot{H}^{s}}=\sqrt{\int_{\mathbb{R}^{n}} \int_{\mathbb{R}^{n}} \frac{|f(x)-f(y)|^{2}}{|x-y|^{n+2 s}} \mathrm{~d} x \mathrm{~d} y .}
$$

That is equivalent to

$$
\|f\|_{\dot{H}^{s}} \cong \sqrt{\int_{\mathbb{R}^{n}}|\xi|^{2 s}|\hat{f}(\xi)|^{2} \mathrm{~d} \xi} .
$$

In some texts, this space $\dot{H}^{s}$ is written as $\dot{L}^{2 s, 2}$.

When $n-2 s>0$, the Sobolev embedding results say that $\dot{H}^{s} \subset L^{2 n /(n-2 s)}$ (see, for example, [14, chap. V]). The space $\dot{H}^{s}$ is defined as the completion of $\mathcal{S}$ with the norm $\|\cdot\|_{\dot{H}^{s}}$. Indeed, $\dot{H}^{s}$ is the space of $L^{2 n /(n-2 s)}$ functions for which (3.2) is integrable. The space $\dot{H}^{s}$ is a Hilbert space with the inner product given by

$$
\begin{aligned}
\langle f, g\rangle_{\dot{H}^{s}} & =\int_{\mathbb{R}^{n}} \int_{\mathbb{R}^{n}} \frac{(f(x)-f(y))(g(x)-g(y))}{|x-y|^{n+2 s}} \mathrm{~d} x \mathrm{~d} y \\
& =\int_{\mathbb{R}^{n}} f(x)(-\triangle)^{s} g(x) \mathrm{d} x \\
& \simeq \int_{\mathbb{R}^{n}}|\xi|^{2 s} \hat{f}(\xi) \overline{\hat{g}(\xi)} \mathrm{d} \xi .
\end{aligned}
$$

The set $\left\{u \in \dot{H}^{s}: \varphi \leq u\right\}$ is clearly convex and closed, and it is easy to show that it is nonempty because $\varphi$ is bounded and has compact support. Thus, the (strictly convex) functional $J$ has a unique minimum in that set. Let $u$ be this minimizer. In the following propositions, we will prove that $u$ is a solution of our obstacle problem.

PROPOSITION 3.1 The function $u$ is a supersolution of $(-\triangle)^{s} u \geq 0$.

PROOF: Let $h$ be any smooth nonnegative function with compact support, and let $t>0$. The function $u+t h$ is above the obstacle, and so $\|u+t h\|_{\dot{H}^{s}} \geq\|u\|_{\dot{H}^{s}}$. 
Therefore

$$
\begin{aligned}
\langle u, u\rangle_{\dot{H}^{s}} & \leq\langle u+t h, u+t h\rangle_{\dot{H}^{s}}, \\
0 & \leq t\langle u, h\rangle_{\dot{H}^{s}}+t^{2}\langle h, h\rangle_{\dot{H}^{s}} \\
& \leq t\left\langle u,(-\triangle)^{s} h\right\rangle_{L^{2}}+t^{2}\langle h, h\rangle_{\dot{H}^{s}} \\
& \leq t \int u(-\triangle)^{s} h \mathrm{~d} x+t^{2}\langle h, h\rangle_{\dot{H}^{s}} .
\end{aligned}
$$

Letting $t \rightarrow 0^{+}$, we get that $\int u(-\triangle)^{s} h \mathrm{~d} x=\int\left((-\triangle)^{s} u\right) h \mathrm{~d} x \geq 0$ for any nonnegative test function $h$. Therefore $(-\triangle)^{s} u$ is a nonnegative measure.

COROLlARY 3.2 The function $u$ is lower-semicontinuous and the set $\{u>\varphi\}$ is open.

Proof: Since $(-\triangle)^{s} u \geq 0$, by Proposition $2.15 u$ is lower-semicontinuous. Thus $\{u>\varphi\}$ is open.

Proposition 3.3 Let $x_{0} \in \mathbb{R}^{n}$ such that $u\left(x_{0}\right)>\varphi\left(x_{0}\right)$. Let $r>0$ such that $u>\varphi$ in $B_{r}\left(x_{0}\right)$; then $(-\triangle)^{s} u\left(x_{0}\right)=0$ in $B_{r}\left(x_{0}\right)$.

PROOF: Since $u$ is lower-semicontinuous, the infimum of $u-\varphi$ is achieved in $B_{r}\left(x_{0}\right)$; then there is an $\varepsilon>0$ such that $u>\varphi+\varepsilon$ in $B_{r}\left(x_{0}\right)$. For any continuous function $h$ supported in $B_{r}\left(x_{0}\right), u+t h$ is going to be above $\varphi$ for $t$ small enough. So the same computation as in the proof of Proposition 3.1 takes place. But this time $t$ and $h$ do not need to be nonnegative, and $(-\triangle)^{s} u=0$ is obtained in $B_{r}\left(x_{0}\right)$.

The following proposition is a modification of a theorem of Evans for superharmonic functions. It will be used to prove continuity for $u$.

Proposition 3.4 Let $v$ be a bounded function in $\mathbb{R}^{n}$ such that $(-\triangle)^{\sigma} v \geq 0$ and $\left.v\right|_{E}$ is continuous where $E=\operatorname{supp}(-\triangle)^{\sigma} v$. Then $v$ is continuous in $\mathbb{R}^{n}$.

PROOF: In the open set $\mathbb{R}^{n} \backslash E,(-\triangle)^{\sigma} v=0$, and thus $v$ is continuous there. We are left to check that $v$ is continuous in $E$.

Let $x_{0} \in E$ and $x_{k} \rightarrow x_{0}$. Since $v$ is lower-semicontinuous, $\liminf _{k \rightarrow \infty} v\left(x_{k}\right) \geq$ $v\left(x_{0}\right)$. We are left to prove $\lim \sup _{k \rightarrow \infty} v\left(x_{k}\right) \leq v\left(x_{0}\right)$. Suppose the contrary; then we can extract a subsequence such that

$$
\lim _{k \rightarrow \infty} v\left(x_{k}\right)=v\left(x_{0}\right)+a
$$

where $a>0$. Since $\left.v\right|_{E}$ is continuous, then $x_{k} \notin E$ from some $k$ on. We can assume that $x_{k} \notin E$ for any $k$ by dropping the first few elements in the sequence.

Let $y_{k}$ be the point in $E$ closest to $x_{k}$ (or one of them). Since $v$ is continuous in $E, \lim v\left(y_{k}\right)=v\left(x_{0}\right)$. Let $\lambda_{k}=\left|x_{k}-y_{k}\right|=\operatorname{dist}\left(x_{k}, E\right)$, so $\lambda_{k} \rightarrow 0$ as $k \rightarrow 0$. Let 
$c_{0}=\inf \left(\gamma_{1}(x+e)\right) /\left(\gamma_{1}(x)\right)$, where $e$ is any unit vector. The infimum is achieved at one point, and thus $c_{0}>0$, since

$$
\lim _{|x| \rightarrow \infty} \frac{\gamma_{1}(x+e)}{\gamma_{1}(x)}=1
$$

By symmetry, the value of $c_{0}$ does not depend on $e$. Setting $e=\left(x_{k}-y_{k}\right) / \lambda_{k}$, we have

$$
\begin{aligned}
\gamma_{\lambda_{k}}\left(x-y_{k}\right)-c_{0} \gamma_{\lambda_{k}}\left(x-x_{k}\right) & =\frac{1}{\lambda_{k}^{n}}\left(\gamma_{1}\left(\frac{x-x_{k}}{\lambda_{k}}+\frac{x_{k}-y_{k}}{\lambda_{k}}\right)-c_{0} \gamma_{1}\left(\frac{x-x_{k}}{\lambda_{k}}\right)\right) \\
& \geq 0 .
\end{aligned}
$$

We now use Proposition 2.15 for $v\left(y_{k}\right)$ and Proposition 2.22 for $v\left(x_{k}\right)$ to get

$$
\begin{aligned}
v\left(y_{k}\right) \geq & \int_{\mathbb{R}^{n}} \gamma_{\lambda_{k}}\left(x-y_{k}\right) v(x) \mathrm{d} x \\
\geq & \int_{\mathbb{R}^{n}} c_{0} \gamma_{\lambda_{k}}\left(x-x_{k}\right) v(x) \mathrm{d} x \\
& +\int_{\mathbb{R}^{n}}\left(\gamma_{\lambda_{k}}\left(x-y_{k}\right)-c_{0} \gamma_{\lambda_{k}}\left(x-x_{k}\right)\right) v(x) \mathrm{d} x \\
\geq & c_{0} v\left(x_{k}\right)+\mathrm{I}_{1}+\mathrm{I}_{2}
\end{aligned}
$$

where

$$
\begin{aligned}
& \mathrm{I}_{1}=\int_{B \sqrt{\lambda_{k}}\left(y_{k}\right)}\left(\gamma_{\lambda_{k}}\left(x-y_{k}\right)-c_{0} \gamma_{\lambda_{k}}\left(x-x_{k}\right)\right) v(x) \mathrm{d} x, \\
& \mathrm{I}_{2}=\int_{\mathbb{R}^{n} \backslash B \sqrt{\lambda_{k}}\left(y_{k}\right)}\left(\gamma_{\lambda_{k}}\left(x-y_{k}\right)-c_{0} \gamma_{\lambda_{k}}\left(x-x_{k}\right)\right) v(x) \mathrm{d} x .
\end{aligned}
$$

Since $v$ is lower-semicontinuous, when $k \rightarrow \infty, \lambda_{k} \rightarrow 0$, and $v\left(x+y_{k}\right) \geq$ $v\left(x_{0}\right)-\varepsilon_{k}$ for any $x \in B_{\sqrt{\lambda_{k}}\left(y_{k}\right)}$ and $\varepsilon_{k} \rightarrow 0$. Moreover, recalling that $\gamma_{\lambda}(x)=$ $\gamma_{1}(x / \lambda) / \lambda^{n}$ and $\int \gamma_{1} \mathrm{~d} x=1$, if we set $z=\left(x-x_{k}\right) / \lambda_{k}$ and $e=\left(y_{k}-x_{k}\right) / \lambda_{k}$,

$$
\begin{aligned}
\int_{B_{\sqrt{\lambda}}\left(y_{k}\right)}\left(\gamma_{\lambda_{k}}\left(x-y_{k}\right)-c_{0} \gamma_{\lambda_{k}}\left(x-x_{k}\right)\right) \mathrm{d} x & =\int_{B_{\lambda^{-1 / 2}}}\left(\gamma_{1}(z)-c_{0} \gamma_{1}(z+e)\right) \mathrm{d} z \\
& \geq 1-c_{0}-\tilde{\varepsilon}_{k}
\end{aligned}
$$

for $\tilde{\varepsilon}_{k} \rightarrow 0$ as $k \rightarrow \infty$. Combining these last two facts, we obtain (3.7) $\mathrm{I}_{1} \geq\left(1-c_{0}-\varepsilon_{k}\right)\left(v\left(x_{0}\right)-\varepsilon_{k}\right)=\left(1-c_{0}\right) v\left(x_{0}\right)-\tilde{\varepsilon}_{k} v\left(x_{0}\right)+\varepsilon_{k}\left(1-c_{0}-\tilde{\varepsilon}_{k}\right)$. 
Now we estimate $\mathrm{I}_{2}$,

$$
\mathrm{I}_{2} \geq-\|v\|_{L^{\infty}} \int_{\mathbb{R}^{n} \backslash B_{\sqrt{\lambda}}\left(y_{k}\right)}\left(\gamma_{\lambda_{k}}\left(x-y_{k}\right)-c_{0} \gamma_{\lambda_{k}}\left(x-x_{k}\right)\right) \mathrm{d} x \geq-\tilde{\varepsilon}_{k}\|v\|_{L^{\infty}} .
$$

Substituting (3.7) and (3.8) in (3.4), we get

$$
v\left(y_{k}\right) \geq c_{0} v\left(x_{k}\right)+\left(1-c_{0}\right) v\left(x_{0}\right)-2 \tilde{\varepsilon}_{k}\|v\|_{L^{\infty}}+\varepsilon_{k}\left(1-c_{0}-\tilde{\varepsilon}_{k}\right),
$$

where $\varepsilon_{k}$ and $\tilde{\varepsilon}_{k}$ go to 0 as $k \rightarrow \infty$. But this is a contradiction since $v\left(y_{k}\right) \rightarrow v\left(x_{0}\right)$ and $v\left(x_{k}\right) \rightarrow v\left(x_{0}\right)+a$ as $k \rightarrow \infty$.

COROLlary 3.5 The function $u$ is continuous.

In this way we finished proving that the minimizer of the functional $J(u)$ solves the original obstacle problem (1.1)-(1.4).

\subsection{Semiconvexity}

We are going to show that when $\varphi$ is smooth enough, the solution $u$ to our obstacle problem is Lipschitz and semiconvex. When $\varphi$ has weaker smoothness assumptions, we will get correspondingly weaker conditions for $u$. The proofs in this subsection depend only on the maximum principle and translation invariance. This regularity is common for all obstacle problems with operators satisfying these two conditions.

PROPOSITION 3.6 The function $u$ is the least supersolution of $(-\triangle)^{s} u \geq 0$ that is above $\varphi(u \geq \varphi)$ and is nonnegative at infinity $\left(\liminf _{|x| \rightarrow+\infty} u(x) \geq 0\right)$.

PROOF: Let $v$ be another supersolution of $(-\triangle)^{s} v \geq 0$ such that $v \geq \varphi$ and $\liminf _{|x| \rightarrow+\infty} v(x) \geq 0$. Let $m=\min (u, v)$. We want to show that actually $m=u$. By definition $m \leq u$, we are left to show $m \geq u$.

Since both $u$ and $v$ are supersolutions, by Proposition 2.18, so is $m$. The function $m$ is also above $\varphi$ because both $u$ and $v$ are; then $m$ is another supersolution that is above $\varphi$. By Proposition 2.17, $m$ is lower-semicontinuous in $\mathbb{R}^{n}$.

Since $\varphi \leq m \leq u$, then $\lim _{x \rightarrow \infty} m(x)=0$. For every $x$ in the contact set $\{u=\varphi\}, m(x)=u(x)$. In $\Omega=\{u>\varphi\}, u$ is a solution of $(-\triangle)^{s} u=0$ and $m$ a supersolution. By Corollary $3.5 u$ is continuous; then $m-u$ is lowersemicontinuous. Then $m \geq u$ by the comparison principle (Proposition 2.21).

COROLlary 3.7 The function $u$ is bounded and $\sup u \leq \sup \varphi$.

Proof: By hypothesis $u \geq 0$. The constant function $v(x)=\sup \varphi$ is a supersolution that is above $\varphi$. By Proposition 3.6, $u \leq v$ in $\mathbb{R}^{n}$.

THEOREM 3.8 If the obstacle $\varphi$ has a modulus of continuity $c$, then the function $u$ also has the same modulus of continuity. 
Proof: Since $c$ is a modulus of continuity for $\varphi$, for any $h \in \mathbb{R}^{n}, \varphi(x+h)+$ $c(|h|) \geq \varphi(x)$ for every $x \in \mathbb{R}^{n}$. Then the function $u(x+h)+c(|h|)$ is also a supersolution above $\varphi$. By Proposition 3.6, $u(x+h)+c(|h|) \geq u(x)$ for any $x, h \in \mathbb{R}^{n}$. Thus $u$ also has $c$ as a modulus of continuity.

COROLlaRY 3.9 The function $u$ is Lipschitz, and its Lipschitz constant is not larger than the one for $\varphi$.

PROOF: It follows from Proposition 3.8 with $c(r)=C r$.

Proposition 3.10 Suppose that $\varphi \in C^{1,1}$. For any vector $e \in \mathbb{R}^{n}$, let $C=$ $\sup -\partial_{e e} \varphi$. Then $\partial_{e e} u \geq-C$ too. Thus, $u$ is semiconvex, and therefore for any point $x \in \mathbb{R}^{n}$, there is a paraboloid of opening $C$ touching $u$ from below.

PROOF: Since $\partial_{e e} \varphi \geq-C$, we look at the second incremental quotients:

$$
\frac{\varphi(x+t e)+\varphi(x-t e)}{2}+C t^{2} \geq \varphi
$$

for every $t>0$ and $x \in \mathbb{R}^{n}$. Therefore

$$
v(x):=\frac{u(x+t e)+u(x-t e)}{2}+C t^{2} \geq \varphi,
$$

and $v$ is also a supersolution of $(-\triangle)^{s} v \geq 0$. By Proposition 3.6, $v \geq u$; then

$$
v(x)=\frac{u(x+t e)+u(x-t e)}{2}+C t^{2} \geq u(x)
$$

for every $t>0$ and $x \in \mathbb{R}^{n}$. Therefore $\partial_{e e} u \geq-C$.

The above proposition is enough to treat the case when $\varphi$ is $C^{1,1}$. However, to obtain the sharp estimates for $\varphi \in C^{1, \alpha}$, we need to refine the previous result. The following propositions are proven more or less with the same idea used in Proposition 3.10, but with an incremental expression for the fractional Laplacian instead of the second-order incremental quotient. The reader interested only in the case when $\varphi \in C^{1,1}$ can jump to Proposition 3.16.

Proposition 3.11 Let us suppose that $(-\triangle)^{\sigma} \varphi \leq C$ for some constant $C$ and some $\sigma \in(0,1)$. Then also $(-\triangle)^{\sigma} u \leq C$ (maybe for another $C$ depending on the dimension $n)$.

Proof: We apply Corollary 2.20 instead of the second-order incremental quotient to obtain

$$
\varphi(x) \leq \varphi * \gamma_{\lambda}(x)+C \lambda^{2 \sigma}
$$

for any $x \in \mathbb{R}^{n}$ and any $\lambda$. Since $(-\triangle)^{s}\left(u * \gamma_{\lambda}+C \lambda^{2 \sigma}\right)=(-\triangle)^{s} u * \gamma_{\lambda} \geq 0$ and

$$
u * \gamma_{\lambda}+C \lambda^{2 \sigma} \geq \varphi * \gamma_{\lambda}+C \lambda^{2 \sigma} \geq \varphi(x),
$$

then $u * \gamma_{\lambda}+C \lambda^{2 \sigma} \geq u$ by Proposition 3.6. Thus $(-\triangle)^{s} u \geq-C$ by Corollary 2.16 .

Proposition 3.12 If $(-\triangle)^{s} \varphi \in L^{\infty}\left(\mathbb{R}^{n}\right)$, then $(-\triangle)^{s} u \in L^{\infty}\left(\mathbb{R}^{n}\right)$. 
ProOF: This proposition follows by combining Proposition 3.11 with the fact that $(-\triangle)^{s} u \geq 0$.

PROpOSITION 3.13 If $\varphi \in C^{1, \alpha}$, then for every $x_{0} \in \mathbb{R}^{n}$, there is a vector a $\in \mathbb{R}^{n}$ such that

$$
u\left(x_{0}+h\right) \geq u\left(x_{0}\right)+a \cdot h-C|h|^{1+\alpha} \quad \text { for every } h \in \mathbb{R}^{n} ;
$$

i.e., the function $u$ has a supporting plane at each point with an error of order $1+\alpha$.

To prove this proposition we will need a couple of lemmas.

Lemma 3.14 Suppose $\varphi \in C^{1+\alpha}$. If $0=\sum \lambda_{j} h_{j}$ for $h_{j} \in \mathbb{R}^{n}$ and $\lambda_{j} \in[0,1]$ such that $\sum \lambda_{j}=1$, then

$$
u(x) \leq \sum \lambda_{j} u\left(x+h_{j}\right)+C \sum \lambda_{j}\left|h_{j}\right|^{1+\alpha} \quad \text { for any } x \in \mathbb{R}^{n} .
$$

Proof: Since $\varphi \in C^{1+\alpha}$, there is a constant $C$ for which

$$
\begin{aligned}
\sum \lambda_{j} \varphi\left(x+h_{j}\right) & +C \sum \lambda_{j}\left|h_{j}\right|^{1+\alpha} \\
& \geq \sum \lambda_{j}\left(\varphi(x)+\nabla \varphi(x) \cdot h_{j}-C\left|h_{j}\right|^{1+\alpha}\right)+C \sum \lambda_{j}\left|h_{j}\right|^{1+\alpha} \\
& \geq \varphi(x)+\nabla \varphi(x) \cdot \sum \lambda_{j} h_{j}=\varphi(x) .
\end{aligned}
$$

As in the proof of Proposition 3.10, we obtain that

$$
v(x)=\sum \lambda_{j} u\left(x+h_{j}\right)+C \sum \lambda_{j}\left|h_{j}\right|^{1+\alpha}
$$

is a function above $\varphi$ such that $(-\triangle)^{s} v \geq 0$, and thus it is also above $u$. By Proposition 3.6,

$$
\sum \lambda_{j} u\left(x+h_{j}\right)+C \sum \lambda_{j}\left|h_{j}\right|^{1+\alpha} \geq u(x),
$$

as we wanted to show.

LEMMA 3.15 Let $f: \mathbb{R} \rightarrow \mathbb{R}$ be a Lipschitz function that satisfies an inequality like (3.9). Then for every $x \in \mathbb{R}, f$ has right and left derivatives at $x$. Moreover, the right derivative is greater than the left derivative, and for any number $a$ in the closed interval between them and for every $h \in \mathbb{R}$,

$$
f(x+h) \geq f(x)+a \cdot h-C|h|^{1+\alpha}
$$

where C depends only on the constant of inequality (3.9).

Proof: We will show that $f$ has derivatives from both sides, and the one from the left is smaller than or equal to the one from the right. Then we will show that (3.10) holds for any $a$ between the two derivatives. 
Let $x_{0}$ be any point in $\mathbb{R}$, and $0<h^{\prime}<h$. Consider the inequality (3.9) with $x=x_{0}+h^{\prime}, h_{1}=-h^{\prime}$, and $h_{2}=h-h^{\prime}$, and thus $\lambda_{1}=\left(h-h^{\prime}\right) / h$ and $\lambda_{2}=h^{\prime} / h$. We have

$$
\begin{aligned}
f\left(x_{0}+h^{\prime}\right) \leq & \frac{h-h^{\prime}}{h} f\left(x_{0}\right)+\frac{h^{\prime}}{h} f(x+h) \\
& +C\left(\frac{h-h^{\prime}}{h}\left|h^{\prime}\right|^{1+\alpha}+\frac{h^{\prime}}{h}\left|h-h^{\prime}\right|^{1+\alpha}\right) \\
\leq & f(x)+\frac{h^{\prime}}{h}(f(x+h)-f(h))+C h^{\prime} \cdot h^{\alpha} .
\end{aligned}
$$

Then,

$$
\frac{f\left(x+h^{\prime}\right)-f(x)}{h^{\prime}} \leq \frac{f(x+h)-f(x)}{h}+C h^{\alpha} .
$$

Since $f$ is Lipschitz, its incremental quotients are bounded, and then (3.11) implies that $(f(x+h)-f(x)) / h$ has a limit as $h \rightarrow 0^{+}$. Thus the right derivative exists. Similarly, we can show that the left derivative exists.

We want to see now that the right derivative is greater than or equal to the left one. Consider $h_{1}=h$ and $h_{2}=-h$ in inequality (3.9); then

$$
f(x) \leq \frac{1}{2} f(x+h)+\frac{1}{2} f(x-h)+C h^{1+\alpha} .
$$

Therefore,

$$
\frac{f(x)-f(x-h)}{h} \leq \frac{f(x+h)-f(x)}{h}+2 C h^{\alpha} .
$$

Taking $h \rightarrow 0$, we obtain that the right derivative is not less than the left one. Let $a$ be a real number that is not greater than the right derivative and no less than the left derivative. Taking $h^{\prime} \rightarrow 0$ in (3.11),

$$
a \leq \frac{f(x+h)-f(x)}{h}+C h^{\alpha} .
$$

Then

$$
f(x+h) \geq f(x)+a \cdot h-C h^{1+\alpha} .
$$

The result follows similarly for negative $h$.

We are now ready to prove Proposition 3.13.

Proof OF Proposition 3.13: By Lemma 3.14 we have that if $0=\sum \lambda_{j} h_{j}$ for $h_{j} \in \mathbb{R}^{n}$ and $\lambda_{j} \in[0,1]$ such that $\sum \lambda_{j}=1$, then

$$
u(x) \leq \sum \lambda_{j} u\left(x+h_{j}\right)+C \sum \lambda_{j}\left|h_{j}\right|^{1+\alpha} \quad \text { for any } x \in \mathbb{R}^{n} .
$$

Let us assume, without loss of generality, that $x_{0}=0$. For any unit vector $e$, we apply Lemma 3.15 to see that $u$ satisfies the inequality (3.9) in every ray from 
the origin with possibly a different linear part. That means that for each $e \in \mathbb{S}^{n-1}$, there is a real number $a(e)$ such that

$$
u(t e) \geq u(0)+a(e) \cdot t-C|t|^{1+\alpha}
$$

where the constant $C$ does not depend on $e$ and $a(e)$ is the directional derivative

$$
a(e)=\lim _{t \rightarrow 0^{+}} \frac{u(t e)-u(0)}{t} .
$$

Now we will show that the function $c(x)=a(x /|x|) \cdot|x|$, which is homogeneous of degree 1 , is convex. We have to see that

$$
c(\lambda x+(1-\lambda) y) \leq \lambda c(x)+(1-\lambda) c(y) .
$$

We use inequality (3.12) to obtain

$$
u(\lambda t x+(1-\lambda) t y) \leq \lambda u(t x)+(1-\lambda) u(t y)+C(t|x-y|)^{1+\alpha}
$$

for any real number $t$. We now subtract $u(0)$ from both sides and divide by $t$ to obtain

$$
\begin{aligned}
& \frac{u(\lambda t x+(1-\lambda) t y)-u(0)}{t} \\
& \quad \leq \lambda \frac{u(t x)-u(0)}{t}+(1-\lambda) \frac{u(t y)-u(0)}{t}+C t^{\alpha}(|x-y|)^{1+\alpha} .
\end{aligned}
$$

Now we let $t \rightarrow 0$ and replace the limit of the quotients by the corresponding value of $c$ using the directional derivatives of $u$ to obtain

$$
c(\lambda x+(1-\lambda) y) \leq \lambda c(x)+(1-\lambda) c(y) .
$$

Now that we know that $c$ is convex, let $a$ be the slope of a supporting plane at the origin ( $a$ is a vector in the subdifferential of $c$ at 0 ). Then $c(h) \geq a \cdot h$ for every $h \in \mathbb{R}^{n}$. Therefore, recalling (3.13),

$$
u(h) \geq u(0)+c(h)-C|h|^{1+\alpha} \geq u(0)+a \cdot h-C|h|^{1+\alpha},
$$

which concludes the proof.

We finish this section by showing that $u$ solves the third specification of the problem.

PROPOSITION 3.16 For any closed set $\Lambda \subset \mathbb{R}^{n}$, let $v$ be the solution of

- $v(x)=\varphi(x)$ in $\Lambda$,

- $(-\triangle)^{s} v=0$ in $\mathbb{R}^{n} \backslash \Lambda$, and

- $\lim _{|x| \rightarrow \infty} v(x)=0$.

Then $v \leq u$ in $\mathbb{R}^{n}$ (obviously, in the case $\Lambda=\{u=\varphi\}, u=v$ ).

PROOF: Since $u \geq \varphi$ in $\mathbb{R}^{n}$, then $u \geq v$ in $\Lambda$. Since $u$ is a supersolution of $(-\triangle)^{s} u \geq 0$ in $\mathbb{R}^{n} \backslash \Lambda$ and $v$ is a solution, then $u \geq v$ in $\mathbb{R}^{n} \backslash \Lambda$ by the maximum principle. 


\section{An Improvement in Regularity}

In the rest of this work, we will study further regularity results for $u$. This section is devoted to showing that $(-\triangle)^{s} u$ is $C^{\alpha}$ for some small $\alpha$ if the obstacle $\varphi$ is smooth enough.

\subsection{Problem}

To study the regularity of the problem, it is convenient to consider the difference $u-\varphi$. The problem that we obtain is also an obstacle problem; the obstacle is 0 , but there is a right-hand side for the equation. For convenience, we will continue to call $u$ our solution, although the problem is slightly different.

Thus, we have $u$ as a solution to the following free boundary problem:

$$
\begin{aligned}
u(x) & \geq 0, \\
(-\triangle)^{s} u(x) & \geq \phi(x), \\
(-\triangle)^{s} u(x) & =\phi(x) \quad \text { when } u>0,
\end{aligned}
$$

where $\phi$ is $-(-\triangle)^{s} \varphi$ for the obstacle $\varphi$ of the previous sections.

Since we are going to be using $(-\triangle)^{s} u(x)$ a great deal, we define $w=(-\triangle)^{s} u$.

If we assume $\varphi$ to be $C^{\infty}$, the right-hand side $\phi$ will be $C^{\infty}$ as well. However, it will be enough for all the proofs to come (and actually more than enough) to assume $\phi$ to be just Lipschitz.

If we assume $\varphi \in C^{1, \beta}$ for some $\beta \in(0,1)$ such that $1+\beta>2 s$, we will have $\phi \in C^{1+\beta-2 s}$. The results of this section will still apply for this case, but the proofs have some extra complications. For simplicity, we will address this case only at the end of the section. At first we will assume $\varphi$ to be $C^{\infty}$, and thus $\phi$ to be Lipschitz.

In this situation, we know from Section 3 that the function $u$ is a bounded Lipschitz function (Proposition 3.9) and also semiconvex (Proposition 3.10), and so far we know that $w$ is $L^{\infty}$ from Corollary 3.12. We want to prove that $w$ is Hölder-continuous. To summarize, we know:

$$
\begin{aligned}
\sup _{x}|\phi(x)| & \leq C, \\
\sup _{x, y} \frac{|\phi(x)-\phi(y)|}{|x-y|} & \leq C, \\
u_{e e} & \geq-C \text { for every } e \text { such that }|e|=1, \\
|w(x)| & \leq C,
\end{aligned}
$$

for some constant $C$. 


\subsection{A Few Lemmas}

LEMMA 4.1 For $\sigma \in(0,1)$, there is a constant $C$ depending only on $\sigma$ and dimension such that if $v$ is bounded and semiconvex,

$$
\begin{gathered}
\sup _{x}|v(x)| \leq A, \\
\inf _{x|e|=1} \inf _{e e} v_{e e}(x) \geq-B,
\end{gathered}
$$

then $\sup _{x}(-\triangle)^{\sigma} v(x) \leq C \cdot B^{\sigma} \cdot A^{1-\sigma}$.

Proof: We can assume that $v$ is smooth. Otherwise, we take a smooth, compactly supported function $\psi$ with integral 1 and consider the approximation of the identity $\psi_{\lambda}(x)=\lambda^{n} \psi(\lambda x)$. Then for any $\lambda>0, \psi_{\lambda} * v$ satisfies (4.8) and (4.9) and is a smooth function. If we can obtain that $\sup _{x}\left|(-\triangle)^{\sigma}\left(\psi_{\lambda} * v\right)(x)\right| \leq C \cdot B^{\sigma} \cdot A^{1-\sigma}$ uniformly in $\lambda$, then we pass to the limit as $\lambda \rightarrow 0$. Therefore, it is enough to prove the lemma for smooth $v$.

The value of $(-\triangle)^{\sigma} v(x)$ can be computed with the integral

$$
\begin{aligned}
(-\triangle)^{\sigma} v(x)= & \int_{\mathbb{R}^{n}} \frac{v(x)-v(y)}{|x-y|^{n+2 \sigma}} \mathrm{d} y \\
\leq & \int_{B_{R}(x)} \frac{v(x)-v(x)-\nabla v(x) \cdot(y-x)+B|y-x|^{2}}{|x-y|^{n+2 \sigma}} \mathrm{d} y \\
& +\int_{\mathbb{R}^{n} \backslash B_{R}(x)} \frac{A}{|x-y|^{n+2 \sigma}} \mathrm{d} y \\
\leq & B\left(\int_{B_{R}(x)} \frac{1}{|x-y|^{n+2 \sigma-2}} \mathrm{~d} y\right)+A\left(\int_{\mathbb{R}^{n} \backslash B_{R}(x)} \frac{1}{|x-y|^{n+2 \sigma}} \mathrm{d} y\right) \\
\leq & C\left(B \cdot R^{2-2 \sigma}+A \cdot R^{-2 \sigma}\right) .
\end{aligned}
$$

Replacing $R$ by $\sqrt{A / B}$ in the above inequality we obtain

$$
(-\triangle)^{\sigma} v(x) \leq C B^{\sigma} A^{1-\sigma} \text {. }
$$

Remark 4.2. Actually in Lemma 4.1, condition (4.9) could be replaced by $\Delta v \geq$ $-B$ by using the fact that

$$
\Delta v=2 n \lim _{r \rightarrow 0^{+}} r^{-2}\left(\frac{1}{\left|\partial B_{r}\right|} \int_{\partial B_{r}} v(y)-v(x) \mathrm{d} y\right) .
$$

LEMMA 4.3 Let $v$ be such that $\left\|(-\triangle)^{\sigma} v(x)\right\|_{\infty} \leq C$. For $\alpha<2 \sigma$, consider $v_{\lambda}=v(\lambda x) / \lambda^{\alpha}$. Then $\left\|(-\triangle)^{\sigma} v_{\lambda}(x)\right\|_{\infty} \rightarrow 0$ as $\lambda \rightarrow 0$. 
PROOF: Recalling that $(-\triangle)^{\sigma}(v(\lambda x))=\lambda^{2 \sigma}\left((-\triangle)^{\sigma} v\right)(\lambda x)$,

$$
\begin{aligned}
\left|(-\triangle)^{\sigma} v_{\lambda}(x)\right| & =\left|\lambda^{-\alpha}\right| \lambda^{2 \sigma}\left((-\triangle)^{2 \sigma} v\right)(\lambda x) \\
& \leq C \lambda^{2 \sigma-\alpha} \rightarrow 0 .
\end{aligned}
$$

LEMMA 4.4 For any $\sigma \in(0,1)$ and $\delta>0$, if $\varepsilon$ and $\alpha$ are chosen small enough, then there is $a \gamma>0$ such that if

$$
\begin{aligned}
& (-\triangle)^{\sigma} v(x) \leq \varepsilon \quad \text { for } x \in B_{1}, \\
& v(x) \leq 1 \quad \text { for } x \in B_{1}, \\
& v(x) \leq|2 x|^{\alpha} \quad \text { for } x \in \mathbb{R}^{n} \backslash B_{1} \text {, } \\
& \delta \leq\left|\left\{x \in B_{1}: v(x) \leq 0\right\}\right|,
\end{aligned}
$$

then $v(x) \leq 1-\gamma$ for $x \in B_{1 / 2}$.

PROOF: As in Lemma 4.1, we can assume $v$ to be smooth.

Let $b(x)=\beta(|x|)$ be a fixed, smooth radial function with support in $B_{1}$ such that $\beta(0)=1$ and $\beta$ is monotone decreasing.

For small enough $\varepsilon$ and $\alpha$, we can choose a positive number $\kappa<\frac{1}{2}$ such that

$$
\varepsilon+\kappa \sup _{x}(-\triangle)^{\sigma} b(x)+\int_{\mathbb{R}^{n}-B_{1 / 4}} \frac{|4 y|^{\alpha}-1}{|y|^{n+2 \sigma}} \mathrm{d} y<\frac{\delta}{2 \cdot 2^{n+2 \sigma}} .
$$

Let $\gamma=\kappa\left(\beta\left(\frac{1}{2}\right)-\beta\left(\frac{3}{4}\right)\right)$. Suppose there is a point $x_{0} \in B_{1 / 2}$ such that $v\left(x_{0}\right)>$ $1-\gamma=1-\kappa \beta\left(\frac{1}{2}\right)+\kappa \beta\left(\frac{3}{4}\right)$. Then $v\left(x_{0}\right)+\kappa b\left(x_{0}\right) \geq 1+\kappa \beta\left(\frac{3}{4}\right)$, which is larger than $v(y)+\kappa b(y)$ for any $y \in B_{1} \backslash B_{3 / 4}$. This means that the supremum of $v(x)+\kappa b(x)$ for $x \in B_{1}$ is greater than 1 and is achieved at an interior point of $B_{3 / 4}$. Let us call that point $x_{1}$. Now we will evaluate $(-\triangle)^{\sigma}(v+\kappa b)\left(x_{1}\right)$.

On one hand, $(-\triangle)^{\sigma}(v+\kappa b)\left(x_{1}\right)=(-\Delta v)^{\sigma} v\left(x_{1}\right)+\kappa(-\Delta v)^{\sigma} b\left(x_{1}\right) \leq \varepsilon+$ $\kappa(-\triangle v)^{\sigma} b\left(x_{1}\right)$. On the other hand, we have

$$
(-\triangle)^{\sigma}(v+\kappa b)\left(x_{1}\right)=\int_{\mathbb{R}^{n}} \frac{(v+\kappa b)\left(x_{1}\right)-(v+\kappa b)(y)}{\left|x_{1}-y\right|^{n+2 \sigma}} \mathrm{d} y .
$$

For any point $z \in B_{1}$ we know $(v+\kappa b)\left(x_{1}\right) \geq(v+\kappa b)(z)$. Let $A_{0}=\{y \in$ $\left.B_{1} \wedge v(y) \leq 0\right\}$. By assumption $\left|A_{0}\right| \geq \delta$. We use (4.12) and that $\kappa<\frac{1}{2}$ to obtain the lower bound:

$$
\begin{aligned}
(-\triangle)^{\sigma}(v+\kappa b)\left(x_{1}\right) \geq & \int_{y \in \mathbb{R}^{n} \backslash B_{1}} \frac{(v+\kappa b)\left(x_{1}\right)-(v+\kappa b)(y)}{\left|x_{1}-y\right|^{n+2 \sigma}} \mathrm{d} y \\
& +\int_{y \in B_{1}} \frac{(v+\kappa b)\left(x_{1}\right)-(v+\kappa b)(y)}{\left|x_{1}-y\right|^{n+2 \sigma}} \mathrm{d} y
\end{aligned}
$$




$$
\begin{aligned}
& \geq \int_{\mathbb{R}^{n} \backslash B_{1}} \frac{1-|2 y|^{\alpha}}{\left|x_{1}-y\right|^{n+2 \sigma}} \mathrm{d} y+\int_{A_{0}} \frac{1-\kappa}{\left|x_{1}-y\right|^{n+2 \sigma}} \mathrm{d} y \\
& \geq \int_{\mathbb{R}^{n} \backslash B_{1 / 4}} \frac{1-|4 y|^{\alpha}}{|y|^{n+2 \sigma}} \mathrm{d} y+\int_{A_{0}} \frac{1 / 2}{\left|x_{1}-y\right|^{n+2 \sigma}} \mathrm{d} y \\
& \geq \int_{\mathbb{R}^{n}-B_{1 / 4}} \frac{1-|4 y|^{\alpha}}{|y|^{n+2 \sigma}}+\frac{\delta}{2 \cdot 2^{n+2 \sigma}} .
\end{aligned}
$$

Therefore

$$
\varepsilon+\kappa \sup _{x}(-\Delta)^{\sigma} b(x) \geq \int_{\mathbb{R}^{n} \backslash B_{1 / 4}} \frac{1-|4 y|^{\alpha}}{|y|^{n+2 \sigma}} \mathrm{d} y+\frac{\delta}{2 \cdot 2^{n+2 \sigma}} .
$$

But this is a contradiction to (4.14).

Remark 4.5. The proof of Lemma 4.4 can be adapted to a more general family of operators instead of $(-\triangle)^{\sigma}$. In [13], it is used to obtain Hölder estimates for the corresponding equations. The operators for which the result applies include the case

$$
T v(x)=\int_{\mathbb{R}^{n}} a(x, y) \frac{v(x)-v(x+y)}{|y|^{n+2 \sigma(x)}} \mathrm{d} y
$$

for $a$ bounded and symmetric $(a(x, y)=a(x,-y))$ and $0<\sigma_{0} \leq \sigma(x) \leq \sigma_{1}<1$. No modulus of continuity whatsoever is required either for $a$ or $\sigma$. All the details can be found in [13].

COROLlARY 4.6 For any $\sigma \in(0,1)$, if $\varepsilon$ and $\alpha$ are chosen small enough, then there is $a \gamma>0$ such that if

$$
\begin{aligned}
& \left|(-\triangle)^{\sigma} v(x)\right| \leq \varepsilon \quad \text { for } x \in B_{1}, \\
& |v(x)| \leq 1 \quad \text { for } x \in B_{1}, \\
& |v(x)| \leq|2 x|^{\alpha} \quad \text { for } x \in \mathbb{R}^{n} \backslash B_{1},
\end{aligned}
$$

then $\operatorname{osc}_{B_{1 / 2}} v \leq 2-\gamma$.

Proof: Consider the same $\gamma$ as in Lemma 4.4 for $\delta=\left|B_{1}\right| / 2$. Suppose

$$
\left|\left\{x \in B_{1}: v(x) \leq 0\right\}\right| \geq \frac{\left|B_{1}\right|}{2}
$$

otherwise we consider $-v$ instead of $v$. By Lemma 4.4, we get $v(x) \leq 1-\gamma$ for $x \in B_{1 / 2}$; we conclude $\operatorname{osc}_{B_{1 / 2}} v \leq 2-\gamma$.

LEMMA 4.7 For any $\sigma \in(0,1)$ and $\alpha \in(0,2 \sigma)$, if $\delta$ is close to $\left|B_{1}\right|$, then $\varepsilon$ can be chosen small enough so that there is a $\gamma>0$ such that if

$$
\begin{aligned}
(-\triangle)^{\sigma} v(x) & \leq \varepsilon & & \text { for } x \\
v(x) & \leq 1 & \text { for } x & \in B_{1},
\end{aligned}
$$




$$
\begin{aligned}
v(x) & \leq 1+|2 x|^{\alpha} \quad \text { for } x \in \mathbb{R}^{n} \backslash B_{1}, \\
\delta & \leq\left|\left\{x \in B_{1}: v(x) \leq 0\right\}\right|
\end{aligned}
$$

then $v(x) \leq 1-\gamma$ for $x \in B_{1 / 2}$.

PROOF: The proof is the same as in Lemma 4.4 with the only difference that we have to choose $\kappa$ such that

$$
\varepsilon+\kappa \sup _{x}(-\triangle)^{\sigma} b(x)+\int_{\mathbb{R}^{n}-B_{1 / 4}} \frac{|4 y|^{\alpha}}{|y|^{n+2 \sigma}} \mathrm{d} y<\inf _{\substack{A \subset B_{1} \\|A|=\delta}} \int_{A} \frac{1 / 2}{\left|x_{1}-y\right|^{n+2 \sigma}} \mathrm{d} y
$$

for which we need $\delta$ close to $\left|B_{1}\right|$ so that the right-hand side is larger than the last term of the left-hand side.

Remark 4.8. If $\alpha, \sigma, \delta$, and $\varepsilon$ are a combination of constants for which Lemma 4.7 applies, then it also applies for lesser values of $\alpha$. In other words, if it holds for one $\alpha$, then it also holds for any $\alpha$ smaller.

LEMMA 4.9 For any $\sigma \in(0,1)$, let $v$ be a function such that $(-\triangle)^{\sigma} v(x)=0$ for $x$ in some open set $\Omega$. Suppose that

$$
|v(y)-v(x)| \leq c(|x-y|)
$$

for every $x \in \mathbb{R}^{n} \backslash \Omega, y \in \mathbb{R}^{n}$, and some modulus of continuity $c$. Then the same holds for every $x, y \in \mathbb{R}^{n}$.

PROOF: We are left to show (4.15) when $x, y \in \Omega$. The function $v$ is continuous in $\Omega$ because of the equation and in $\mathbb{R}^{n} \backslash \Omega$ because of (4.15), so $v$ is continuous. Let $v_{1}(z)=v(z)-v(z+x-y)$; then $(-\triangle)^{\sigma} v_{1}(z)=0$ for $z \in \Omega \cap(\Omega+y-x)$ and $v_{1}(x) \leq c(|x-y|)$ for $z \notin \Omega \cap(\Omega+y-x)$. By the maximum principle $v_{1}(z) \leq c(|x-y|)$ for every $z \in \mathbb{R}^{n}$; evaluating in $z=y$ we obtain the desired result.

\subsection{Further Regularity}

LEMMA 4.10 Given $\mu>0$ and $u$ satisfying (4.6), if $u(x) \geq \mu r^{2}$ for one $x \in B_{r}$, then

$$
\left|\left\{x \in B_{2 r} \mid: u(x)>0\right\} \geq \delta\right| B_{2 r} \mid
$$

for some $\delta$ depending on $\mu$.

PROOF: We know that $u_{e e} \geq-C$ every time $|e|=1$. In other words, $u$ is semiconvex, and for each point $x$ there is a paraboloid touching $u$ from below:

$$
u(y) \geq u(x)+B \cdot(y-x)-\frac{C}{2}|x-y|^{2}
$$

where $B$ is any vector of the subdifferential of $u(y)+(C / 2)|x-y|^{2}$ at $x$.

Now, let us consider the set

$$
A=\{y: B \cdot(y-x) \geq 0\} \cap B_{(\mu / C)^{1 / 2} r}(x) .
$$


If $y \in B_{(\mu / C)^{1 / 2} r}(x)$, then $(C / 2)|x-y|^{2} \leq(\mu / 2) r^{2}$. If $y \in A$, then

$$
\begin{aligned}
u(y) & \geq u(x)+B \cdot(y-x)-\frac{C}{2}|x-y|^{2} \\
& \geq u(x)-\frac{\mu}{2} r^{2} \\
& \geq \frac{\mu}{2} r^{2}>0 .
\end{aligned}
$$

The set $A$ is half of a ball. If $\mu / C \leq 1$, then $A$ is going to be completely contained in $B_{2 r}$, and we obtain the desired result with

$$
\delta=\frac{1}{2}\left(\frac{\mu}{4 C}\right)^{n / 2} .
$$

Otherwise, we take $A^{\prime}=\{y: B \cdot(y-x) \geq 0\} \cap B_{r}(x)$ instead of $A$, and we obtain the desired result with

$$
\delta=\frac{1}{2}\left(\frac{1}{2}\right)^{n} \text {. }
$$

Remark 4.11. Lemma 4.10, as well as Lemmas 4.4 and 4.7, can be applied to any two balls, one inside the other. The outer radius does not have to be double the inner radius. The lemmas, as stated, imply this by rescaling and a standard covering argument. And the proofs also clearly do not depend on the ratio between the radii. Of course, the constants will vary.

We are now ready to start the proof of $w \in C^{\alpha}$.

THEOREM 4.12 Let $u$ and $w$ be as in (4.1)-(4.7) (recall $0<s<1$ ); then $w$ is $C^{\alpha}$ for a universal $\alpha$, and its $C^{\alpha}$-norm depends on the various constants $C$ of (4.4)-(4.7).

Proof: Let us normalize $u$ and $w$ so that $\|w\|_{L^{\infty}}=1$. We want to show that there is a constant $C_{0} \geq 1$ such that for every $x_{0} \in \mathbb{R}^{n}$ and $k \in \mathbb{N}$,

$$
\underset{B_{2-k}\left(x_{0}\right)}{\operatorname{osc}} w \leq C_{0} 2^{-\alpha k} .
$$

This clearly means that $w$ is $C^{\alpha}$.

We will show by induction that (4.16) holds for every $k$. The induction step works when $k \geq k_{0}$ for a large integer $k_{0}$. Then we can choose a large value for $C_{0}$ so that (4.16) holds for any $k \leq k_{0}$.

We can assume $x_{0}=0$. Let us also assume that $0 \in \operatorname{supp} u$; we will consider the case $x_{0} \notin \operatorname{supp} u$ later. Suppose that (4.16) holds for $k=0,1, \ldots, k_{0}$. Let us prove that it also holds for $k=k_{0}+1$. Let $\delta>0$ be a small number to be determined later. We will prove that

$$
\left|\left\{x \in B_{2^{-k} \mid}: w(x)-\inf _{B_{2}-k_{0}} w \leq \frac{C_{0}}{2} 2^{-\alpha k_{0}}\right\}\right| \geq \delta\left|B_{2^{-k_{0}}}\right| .
$$


But first, let us show how (4.17) implies the inductive step.

Consider

$$
v(x)=2 C_{0}^{-1} 2^{k_{0} \alpha}\left(w\left(2^{-k_{0}} x\right)-\inf _{B_{2}-k_{0}} w\right)-1 .
$$

By (4.6), $-\triangle u \leq C$; thus $(-\triangle)^{1-s} v \leq C 2^{k_{0}(\alpha+2 s-2)}$. Then, if $\alpha$ is chosen smaller than 2-2s and $k_{0}$ is large enough, then $v$ satisfies the hypotheses of Lemma 4.4 with $\sigma=1-s$. Therefore, there is a $\gamma>0$ such that $v(x) \leq 1-\gamma$ for $x \in B_{1 / 2}$. Rescaling back to $w$, we obtain $w(x) \leq C_{0} 2^{-k_{0} \alpha}(1-\gamma / 2)+\inf _{B_{2}-k_{0}} w$ for $x \in$

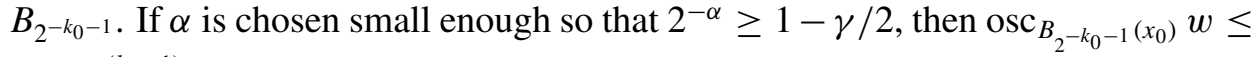
$C_{0} 2^{-\alpha\left(k_{0}+1\right)}$, and the induction step is over.

We are left to prove (4.17). Suppose the contrary:

$$
\left|\left\{x \in B_{2^{-k}}: w(x)-\inf _{B_{2^{-k_{0}}}} w \leq \frac{C_{0}}{2} 2^{-\alpha k_{0}}\right\}\right| \leq \delta\left|B_{2^{-k_{0}}}\right| .
$$

We know $w(x) \geq \phi(x)$ for every $x$. Then $\inf _{B_{2}-k_{0}} w \geq \inf _{B_{2}-k_{0}} \phi$, and since $\phi$ is Lipschitz,

$$
\underset{B_{2}-k_{0}\left(x_{0}\right)}{\operatorname{osc}} \phi \leq C 2^{-k_{0}}<\frac{C_{0}}{2} 2^{-\alpha k_{0}}
$$

for $k_{0}$ large enough.

Every time $u(x)>0, w(x)=\phi(x)$; therefore

$$
\left\{x \in B_{2^{-k}}: u(x)>0\right\} \subset\left\{x \in B_{2^{-k}}: w(x)-\inf _{B_{2^{-k}}} w \leq \frac{C_{0}}{2} 2^{-\alpha k_{0}}\right\}
$$

Thus

$$
\left|\left\{x \in B_{2^{-k}}: u(x)>0\right\}\right| \leq \delta\left|B_{2^{-k_{0}}}\right| .
$$

We choose $\delta$ in this proof to be small, so that the contrareciprocal of Lemma 4.10 applies and we have $u(x) \leq \mu 2^{-2 k_{0}}$ for every $x \in B_{(3 / 4) 2^{-k_{0}}}$.

Let us consider the rescaled problem:

$$
\begin{aligned}
& \bar{u}(x)=C_{0}^{-1} 2^{k_{0}(\alpha+2 s)} u\left(2^{-k_{0}} x\right), \\
& \bar{w}(x)=C_{0}^{-1} 2^{k_{0} \alpha} w\left(2^{-k_{0}} x\right), \\
& \bar{\phi}(x)=C_{0}^{-1} 2^{k_{0} \alpha} \phi\left(2^{-k_{0}} x\right) .
\end{aligned}
$$

The pair $\bar{u}$ and $\bar{w}$ also satisfy the original hypotheses:

$$
\begin{aligned}
\bar{w}(x) & =(-\triangle)^{s} \bar{u}(x), \\
\bar{u}(x) & \geq 0, \\
\bar{w}(x) & \geq \bar{\phi}(x), \\
\bar{w}(x) & =\bar{\phi}(x) \quad \text { when } \bar{u}>0 .
\end{aligned}
$$


From (4.4)-(4.7),

$$
\begin{aligned}
\sup _{x, y} \frac{|\bar{\phi}(x)-\bar{\phi}(y)|}{|x-y|} & \leq C 2^{-k_{0}(1-\alpha)} \\
\bar{u}_{e e} & \geq-C 2^{-k_{0}(2-2 s-\alpha)} \quad \text { for every } e \text { such that }|e|=1 .
\end{aligned}
$$

Also, $\bar{u}(x) \leq C 2^{-k_{0}(2-2 s-\alpha)}$ for every $x \in B_{3 / 4}$.

If $k_{0}$ is large enough, then

$$
\begin{array}{rlrl}
\sup _{x, y} \frac{|\bar{\phi}(x)-\bar{\phi}(y)|}{|x-y|} & \leq \varepsilon, & \\
\bar{u}_{e e} & \geq-\varepsilon & & \text { for every } e \text { such that }|e|=1, \\
0 \leq \bar{u}(x) & \leq \varepsilon & & \text { for every } x \in B_{3 / 4},
\end{array}
$$

for arbitrarily small $\varepsilon$. We choose $\varepsilon$ much smaller than $\delta$. From (4.21) and (4.22), we conclude that $u$ is Lipschitz in $B_{5 / 8}$ and its norm is less than $C \varepsilon$.

The inductive hypothesis, rescaled, turns into

$$
\underset{B_{2^{k}}}{\operatorname{Oosc}} \bar{w} \leq 2^{k \alpha}
$$

for $k=0,1,2, \ldots$

Then

$$
\begin{array}{ll}
|\bar{w}(x)-\bar{w}(0)| \leq|2 x|^{\alpha} & \text { for }|x|>1, \\
|\bar{w}(x)-\bar{w}(0)| \leq 1 & \text { for }|x| \leq 1 .
\end{array}
$$

We also know from (4.19) that

$$
\left|\left\{x \in B_{1}: \bar{w}(x)-\inf _{B_{1}} \bar{w}>\frac{1}{2}\right\}\right| \geq(1-\delta)\left|B_{1}\right| .
$$

Now, let $b$ be a smooth cutoff function such that

$$
\begin{array}{ll}
b(x)=0 & \text { for } x \in \mathbb{R}^{n} \backslash B_{5 / 8} \\
b(x) \equiv 1 & \text { for } x \in B_{7 / 16} \\
b(x) \leq 1 & \text { for every } x \in \mathbb{R}^{n} .
\end{array}
$$

Thus

$$
\begin{aligned}
b(x) \bar{u}(x) & \leq \varepsilon, \\
(b \bar{u})_{e e} & =b_{e e} \bar{u}+2 b_{e} \bar{u}_{e}+b \bar{u}_{e e} \geq-C \varepsilon .
\end{aligned}
$$

Let $h=(-\triangle)^{s}(b \bar{u})$. We can apply Lemma 4.1 to obtain $h \leq C \varepsilon$.

By construction $\bar{u}-b \bar{u} \equiv 0$ in $B_{7 / 16}$, therefore

$$
0=-\triangle(\bar{u}-b \bar{u})=(-\triangle)^{1-s}(\bar{w}-h) \quad \text { in } B_{7 / 16} .
$$


Let $v(x)=1+2\left(h(x)+\inf _{B_{1}} \bar{w}-\bar{w}(x)-C \varepsilon\right)$. Then

$$
\begin{array}{rlrl}
(-\triangle)^{1-s} v & =0 & \\
\sup _{B_{1}} v & \leq 1, & & \text { in } B_{7 / 16}, \\
\sup _{B_{2^{k}}} v & \leq 1+\left(2 \cdot 2^{k}\right)^{\alpha} & & \text { for every positive integer } k, \\
\left|\left\{x \in B_{1}: v(x)<0\right\}\right| & \geq(1-\delta)\left|B_{1}\right| . &
\end{array}
$$

Then, if $\delta$ is chosen small, we can apply Lemma 4.7 (rescaled) to $v$ in the ball $B_{7 / 16}$ to obtain $v(x) \leq 1-\gamma$ for $x \in B_{1 / 2}$, which means

$$
\bar{w}(x) \geq \gamma+\inf _{B_{1}} \bar{w}+h(x)-C \varepsilon
$$

for $x$ in $B_{1 / 2}$.

Let $v_{1}(x)=b(x) \cdot \bar{u}(x)+\varepsilon b(2 x)$. Then $\max v_{1}(x)=v_{1}\left(x_{0}\right)$ for some $x_{0} \in B_{1 / 2}$. Moreover, since $0 \in \operatorname{supp} \bar{u}$ and $\varepsilon b(2 x)$ constantly achieves its maximum in a neighborhood of 0 , then $\bar{u}\left(x_{0}\right)>0$. Therefore $\bar{w}(x)=\bar{\phi}(x)$ for every $x$ in a neighborhood of $x_{0}$; thus $\bar{w}$ and also $\bar{u}$ are sufficiently smooth at $x_{0}$ so that $(-\triangle)^{s} \bar{u}\left(x_{0}\right)$, as well as $(-\triangle)^{s} v_{1}\left(x_{0}\right)$, can be computed. Since $v_{1}$ achieves its maximum at $x_{0}$, $(-\triangle)^{s} v_{1}\left(x_{0}\right) \geq 0$. Therefore

$$
\begin{aligned}
0 & \leq(-\triangle)^{s} v_{1}\left(x_{0}\right)=h\left(x_{0}\right)+\varepsilon 2^{s}(-\triangle)^{s} b\left(2 x_{0}\right) \\
& \leq h\left(x_{0}\right)+C \varepsilon
\end{aligned}
$$

Replacing in (4.26), we obtain

$$
\bar{w}\left(x_{0}\right) \geq \gamma+\inf _{B_{1}} \bar{w}-C \varepsilon .
$$

But since $x_{0} \in \operatorname{supp} \bar{u}, \bar{w}\left(x_{0}\right)=\bar{\phi}\left(x_{0}\right)$, and $\inf _{B_{1}} \bar{w} \geq \inf _{B_{1}} \bar{\phi}$, then

$$
\gamma \leq \bar{\phi}\left(x_{0}\right)-\inf _{B_{1}} \bar{\phi}-C \varepsilon \leq C^{\prime} \varepsilon .
$$

But this is a contradiction if we choose the constants so that $\varepsilon$ is much smaller than $\delta$.

This finishes the proof for $x$ in the support of $u$. To extend this modulus of continuity for all $x \in \mathbb{R}^{n}$, we observe that $(-\triangle)^{1-s} w=0$ in the interior of $\{u=0\}$ and apply Lemma 4.9.

\subsection{The Case When $\varphi \in C^{1, \beta}$}

When we assume $\varphi$ to be only $C^{1, \beta}$ in our obstacle problem, we can still obtain the result of Theorem 4.12 when we have $1+\beta>2 s$. In order to show that, we have to improve some of the lemmas. We will explain the modifications in detail now. 
Since we are not assuming $\varphi \in C^{\infty}$, we only have $\phi \in C^{\tau}$ for $\tau=1+\beta-2 s$. And instead of $u_{e e} \geq-C$, we have only a one-sided $C^{1, \beta}$-estimate saying that for each $x \in \mathbb{R}^{n}$, there is a vector $a(x)$ such that

$$
u(y) \geq u(x)+a(x) \cdot(y-x)-C|y-x|^{1+\beta} .
$$

Instead of Lemma 4.1 we will need the following lemma:

LEMma 4.13 For $\sigma \in(0, \beta)$, there is a constant, depending only on $\sigma, \beta$, and dimension, such that if $\sup _{x}|v(x)| \leq A$ and for each $x$ there is a $a(x)$ such that

$$
v(y) \geq v(x)+a(x) \cdot(y-x)-B|y-x|^{1+\beta} \quad \text { for any } y \in \mathbb{R}^{n},
$$

then $(-\triangle)^{\sigma} v(x) \leq C B^{2 \sigma /(1+\beta)} A^{(1+\beta-2 \sigma) /(1+\beta)}$.

PROOF: The proof is very similar to that for Lemma 4.1. We can also assume $v$ to be smooth and do the computations:

$$
\begin{aligned}
(-\triangle)^{\sigma} v(x)= & \int_{\mathbb{R}^{n}} \frac{v(x)-v(y)}{|x-y|^{n+2 \sigma}} \mathrm{d} y \\
\leq & \int_{B_{R}(x)} \frac{v(x)-v(x)-\nabla v(x) \cdot(y-x)+B|y-x|^{1+\beta}}{|x-y|^{n+2 \sigma}} \mathrm{d} y \\
& +\int_{\mathbb{R}^{n} \backslash B_{R}(x)} \frac{A}{|x-y|^{n+2 \sigma}} \mathrm{d} y \\
\leq & B\left(\int_{B_{R}(x)} \frac{1}{|x-y|^{n+2 \sigma-1-\beta}} \mathrm{d} y\right)+A\left(\int_{\mathbb{R}^{n} \backslash B_{R}(x)} \frac{1}{|x-y|^{n+2 \sigma}} \mathrm{d} y\right) \\
\leq & C\left(B \cdot R^{1+\beta-2 \sigma}+A \cdot R^{-2 \sigma}\right) .
\end{aligned}
$$

Replacing $R$ by $(A / B)^{1 /(1+\beta)}$ in the above inequality we obtain

$$
(-\triangle)^{\sigma} v(x) \leq C B^{\frac{2 \sigma}{1+\beta}} A^{\frac{1+\beta-2 \sigma}{1+\beta}} .
$$

Instead of Lemma 4.10, we will use the following lemma, which does not provide an estimate that is as good as before, but it is enough for our purposes.

LEMMA 4.14 Given $\mu>0$ and $u$ satisfying (4.27), if $u(x) \geq \mu r^{1+\beta}$ for one $x \in B_{r}$, then

$$
\left|\left\{x \in B_{2 r}: u(x)>0\right\}\right| \geq \delta\left|B_{2 r}\right|
$$

for some $\delta$ depending on $\mu$.

PROOF: The proof is identical to the one for Lemma 4.10, but every time there is an estimate with a term $|x-y|^{2},|x-y|^{1+\beta}$ has to be used instead. 
A new lemma has to be added to replace the well-known fact that semiconvex functions are locally Lipschitz.

LEMMA 4.15 Suppose

$$
\begin{aligned}
|u(x)| & \leq \varepsilon & & \text { for } x \in B_{1}, \\
u(y) & \geq u(x)+a(x) \cdot(y-x)-\varepsilon|y-x|^{1+\beta} & & \text { for } x, y \in B_{1} .
\end{aligned}
$$

Then $u$ is Lipschitz in $B_{1 / 2}$ with a Lipschitz norm no greater than $C \varepsilon$ for a constant $C$.

PROOF: For $x, y \in B_{1 / 2}$, let

$$
K=\frac{|u(y)-u(x)|}{|x-y|} .
$$

Let us assume that $u(y) \geq u(x)$ so that $u(y)=u(x)+K|x-y|$. Let $z \in B_{1}$ be in the line determined by $x$ and $y$ so that $|x-z| \geq|y-z| \geq \frac{1}{2}$. From (4.29), we have

$$
\begin{array}{r}
u(y)-K|x-y|=u(x) \geq u(y)+a(y) \cdot(x-y)-\varepsilon|x-y|^{1+\beta}, \\
u(z) \geq u(y)+a(y) \cdot(z-y)-\varepsilon|z-y|^{1+\beta} .
\end{array}
$$

From (4.30),

$$
a(y) \cdot(y-x) \geq K|x-y|-\varepsilon|x-y|^{1+\beta} .
$$

From (4.31),

$$
\begin{aligned}
u(z) & \geq-\varepsilon+a(y) \cdot(y-x) \frac{|z-x|}{|y-x|}-\varepsilon \\
& \geq a(y) \cdot(y-x) \frac{1}{2|y-x|}-2 \varepsilon \\
& \geq\left(K|x-y|-\varepsilon|x-y|^{1+\beta}\right) \frac{1}{2|y-x|}-2 \varepsilon \\
& \geq \frac{K}{2}-3 \varepsilon .
\end{aligned}
$$

Since $u(z) \leq \varepsilon$ for any $z \in B_{1}$, we have $K<8 \varepsilon$.

Now we are ready to state the theorem. Our assumptions now match what we know in the original obstacle problem when $\varphi \in C^{1, \beta}$.

THEOREM 4.16 Let $u$ and $\phi$ satisfy (4.1), (4.2), and (4.3). Let $w=(-\triangle)^{s} u$ (recall $0<s<1)$. Let us also assume (4.4) and (4.7), and also

$$
\begin{array}{rlrl}
C & \geq \sup _{x, y} \frac{|\phi(x)-\phi(y)|}{|x-y|^{\tau}} & \text { for } \tau=1+\beta-2 s \\
u(y) & \geq u(x)+a(x) \cdot(y-x)-C|x-y|^{1+\beta}, & \\
(-\triangle)^{\sigma} u & \leq C & & \text { for any } \sigma<\frac{1+\beta}{2},
\end{array}
$$


where $\beta$ is a positive real number such that $1+\beta>2$ s. Then $w \in C^{\alpha}$ for an $\alpha>0$ depending only on $s, \beta$, and the dimension $n$.

Remark 4.17. In our original obstacle problem, we have (4.33) from Proposition 3.13 and (4.34) from Proposition 3.11. But actually (4.34) could be deduced from 4.33 and the boundedness of $u$ by Lemma 4.13 .

The proof is essentially the same as the proof of Theorem 4.12 with a few modifications.

First of all, for the function $v$ defined in (4.18), we have to use Lemma 4.4 for a $\sigma$ such that $0<2 \sigma<1+\beta-2 s=\tau$, since it is for those $\sigma$ that we know $(-\triangle)^{\sigma} w=(-\triangle)^{\sigma+s} u \leq C$ and therefore $(-\triangle)^{\sigma} v \leq \varepsilon$ for $k_{0}$ large enough.

Later in the proof, when we construct the rescaled function $\bar{u}$, we will have a different upper bound for $\bar{u}$ in $B_{3 / 4}$ given by Lemma 4.14 instead of 4.10. We have $u(x) \leq \mu 2^{-(1+\beta) k_{0}}$ for $x \in B_{(3 / 4) 2^{-k_{0}}}$, which is enough to obtain $0 \leq \bar{u}(x) \leq \varepsilon$ for $x \in B_{3 / 4}$ since $1+\beta>2 s$.

Instead of (4.20) and (4.21), we have

$$
\begin{aligned}
& \sup _{x, y} \frac{|\bar{\phi}(x)-\bar{\phi}(y)|}{|x-y|^{\tau}} \leq \varepsilon \quad \text { for } \tau=1+\beta-2 s, \\
& \bar{u}(y) \geq \bar{u}(x)+\bar{a}(x) \cdot(y-x)-\varepsilon|y-x|^{1+\beta} .
\end{aligned}
$$

This is the point when we need to use Lemma 4.15 to obtain the Lipschitz bound for $\bar{u}$ in $B_{5 / 8}$ to be less than $C \varepsilon$.

Then the proof follows as in Theorem 4.12 until we have to estimate $(b \bar{u})_{e e}$ from below. Instead, we compute a one-sided $C^{1, \alpha}$-estimate for $(b \bar{u})_{e e}$ from the corresponding one for $\bar{u}$ and the smoothness of $b$. We have that

$$
\begin{aligned}
& b(y) \geq b(x)+b^{\prime}(x) \cdot(y-x)-C|x-y|^{1+\beta}, \\
& \bar{u}(y) \geq \bar{u}(x)+\bar{a}(x) \cdot(y-x)-\varepsilon|y-x|^{1+\beta} .
\end{aligned}
$$

Multiplying both inequalities and recalling that $\|\bar{u}\|_{L i p} \leq C \varepsilon$, we get

$$
b(y) \bar{u}(y) \geq b(x) \bar{u}(x)+A(x) \cdot(y-x)-C \varepsilon|y-x|^{1+\beta}
$$

where $A(x)=\bar{a}(x) b(x)+b^{\prime}(x) \bar{u}(x)$. Then we apply Lemma 4.13 instead of Lemma 4.1 to obtain $h=(-\triangle)^{s}(b \bar{u}) \leq C \varepsilon$.

The rest follows exactly as in the proof of Theorem 4.12.

Remark 4.18. It is interesting to notice the difference with the classical obstacle problem (case $s=1$ ). In that case, the result of Theorem 4.16 is not true since $\Delta u$ has a jump discontinuity across the free boundary. The key point is that when $s<$ 1 , we can use the semiconvexity of $u$ to obtain that $w=(-\triangle)^{s} u$ is a subsolution of $(-\triangle)^{1-s} w \leq C$, and this is an elliptic-like equation in the sense that Lemma 4.4 holds. We are thus using this room that we have between $s$ and 1 to gain some extra regularity. 


\section{Towards Optimal Regularity}

The observation that $(-\triangle)^{1-s} w=0$ in the interior of the contact set $\{u=0\}$ will allow us to estimate its growth in the free boundary by using a few barrier functions carefully. In this way, we will achieve optimal (or almost optimal) regularity results.

\subsection{Barriers}

In [9] a Poisson formula for the balayage problem of $(-\triangle)^{\sigma}$ is proven. The formula says that if $g$ is a continuous function in $\mathbb{R}^{n} \backslash B_{r}$, then there exists a function $u$, continuous in $\mathbb{R}^{n}$, such that $u(x)=g(x)$ for every $|x| \geq r$, and $(-\triangle)^{\sigma} u(x)=0$ for every $|x|<r$. The function $u$ in $B_{r}$ is given by the formula

$$
u(x)=\int_{\mathbb{R}^{n} \backslash B_{r}} P(x, y) g(y) \mathrm{d} y
$$

where

$$
P(x, y)=C_{n, \alpha}\left(\frac{r^{2}-|x|^{2}}{|y|^{2}-r^{2}}\right)^{\sigma} \frac{1}{|x-y|^{n}} .
$$

This is known as the balayage problem in $B_{r}$, and $P$ is its corresponding Poisson function.

We can take $r \rightarrow \infty$ in the above formula to obtain a solution of the balayage problem in the semispace $\left\{x_{n}<0\right\}$. If $g$ is a continuous function in $\left\{x_{n} \geq 0\right\}$, then there is a function $u$, continuous in $\mathbb{R}^{n}$, such that $u(x)=g(x)$ for every $x$ such that $x_{n} \geq 0$ and $(-\triangle)^{\sigma} u(x)=0$ every time $x_{n}<0$. The function $u$ in $\left\{x_{n}<0\right\}$ is given by the formula

$$
u(x)=\int_{\left\{y_{n} \geq 0\right\}} P(x, y) g(y) \mathrm{d} y
$$

where

$$
P(x, y)=C_{n, \alpha}\left(\frac{\left|x_{n}\right|}{\left|y_{n}\right|}\right)^{\sigma} \frac{1}{|x-y|^{n}} .
$$

The constant $C_{n, \alpha}$ is chosen so that

$$
1=\int_{\left\{y_{n} \geq 0\right\}} P(x, y) \mathrm{d} y
$$

for any $x$ (by rescaling, it is not hard to see that the above actually does not depend on $x$ ).

Notice that for each fixed $y, P$ is $C^{\sigma}$ across the boundary $\left\{x_{n}=0\right\}$. We are going to construct barriers now to assure this regularity in several cases. 


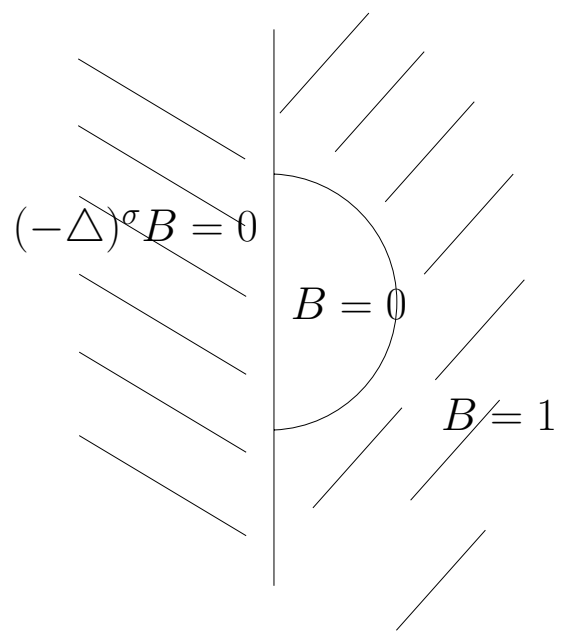

FIGURE 5.1. The function $B$.

Let $g_{0}=1-\chi_{B_{1}}$. When $x_{n} \geq 0$ let $B(x)=g_{0}(x)$, and when $x_{n}<0$ let $B(x)$ be given by the formula

$$
B(x)=\int_{\left\{y_{n} \geq 0\right\}} g_{0}(y) P(x, y) \mathrm{d} y=\int_{\left\{y_{n} \geq 0 \wedge|y| \geq 1\right\}} P(x, y) \mathrm{d} y .
$$

The function $B$ would be the solution of the balayage problem in the semispace $\left\{x_{n}<0\right\}$ for $B(x)=g(x)$ in $\left\{x_{n} \geq 0\right\}$ (see Figure 5.1). In this case $g$ is not continuous, so we just define $B$ as the integral above.

Let us estimate now the behavior of $B$ for small $x$. Let $|x|<\frac{1}{2}$. If $|y|>1$, $\frac{1}{2}|y| \leq|x-y| \leq 2|y| ;$ therefore, since

$$
P(x, y)=C_{n, \alpha}\left(\frac{\left|x_{n}\right|}{\left|y_{n}\right|}\right)^{\sigma} \frac{1}{|x-y|^{n}},
$$

we have

$$
C_{n, \alpha}\left|x_{n}\right|^{\sigma} \frac{1}{2^{n}\left|y_{n}\right|^{\sigma}|y|^{n}} \leq P(x, y) \leq C_{n, \alpha}\left|x_{n}\right|^{\sigma} \frac{2^{n}}{\left|y_{n}\right|^{\sigma}|y|^{n}} .
$$

Applying these estimates to (5.1), we get

$$
\frac{C}{2^{n}}\left|x_{n}\right|^{\sigma} \leq B(x) \leq 2^{n} C\left|x_{n}\right|^{\sigma}
$$

for every $|x|<\frac{1}{2}$, where $C$ depends only on $n$ and $\alpha$ and is given by

$$
C=C_{n, \alpha} \int_{\left\{y_{n} \geq 0 \wedge|y| \geq 1\right\}} \frac{1}{\left|y_{n}\right|^{\sigma}|y|^{n}} \mathrm{~d} y .
$$

On the other hand, when $|x|>1$, it is clear that $1 \geq B(x)>d_{1}$ for some constant $d_{1}>0$ depending only on $\alpha$ and $n$. 
Now let $g_{1}(x)=\min (|x|, 1)$; let us solve the corresponding balayage problem for the semispace $\left\{x_{n}<0\right\}$. We observe that $g_{1}(x)=\int_{0}^{1} g_{0}(x / t) \mathrm{d} t$. Then

$$
A(x)=\int_{\left\{y_{n} \geq 0\right\}} g_{1}(y) P(x, y) \mathrm{d} y=\int_{0}^{1} B\left(\frac{x}{t}\right) \mathrm{d} t .
$$

We want to see the behavior of $A$ when $x$ approaches 0 . Let $|x|<\frac{1}{4}$;

$$
\begin{aligned}
A(x) & =\int_{0}^{1} B\left(\frac{x}{t}\right) \mathrm{d} t=\int_{0}^{2|x|} B\left(\frac{x}{t}\right) \mathrm{d} t+\int_{2|x|}^{1} B\left(\frac{x}{t}\right) \mathrm{d} t \\
& \leq 2|x|+\int_{2|x|}^{1} 2 C\left(\frac{\left|x_{n}\right|}{t}\right)^{\sigma} \mathrm{d} t \\
& \leq 2|x|+\frac{2 C}{r+1}\left|x_{n}\right|^{\sigma}\left(1-(2|x|)^{\sigma}\right) \\
& \leq 2|x|+C\left|x_{n}\right|^{\sigma} .
\end{aligned}
$$

On the other hand,

$$
\begin{aligned}
A(x) & =\int_{0}^{1} B\left(\frac{x}{t}\right) \mathrm{d} t=\int_{0}^{2|x|} B\left(\frac{x}{t}\right) \mathrm{d} t+\int_{2|x|}^{1} B\left(\frac{x}{t}\right) \mathrm{d} t \\
& \geq \int_{0}^{2|x|} B\left(\frac{x}{t}\right) \mathrm{d} t \\
& \geq \int_{0}^{2|x|} d_{1} \mathrm{~d} t=2 d_{1}|x| .
\end{aligned}
$$

The function $A$ is continuous, and clearly $A(x) \rightarrow 1$ as $\left|x_{n}\right| \rightarrow \infty$. Let $\mu=$ $\min _{\mathbb{R}^{n} \backslash B_{1 / 4}} A(x)$. Then

$$
A(x) \geq \min \left(\mu, 2 d_{1}|x|\right) .
$$

If we consider $\tilde{A}(x)=(1 / \mu) A\left(\mu x /\left(2 d_{1}\right)\right)$, then $\tilde{A}(x) \geq \min (1,|x|)$.

PROPOSITION 5.1 Let $v$ be a continuous function in $\mathbb{R}^{n}$ such that

- $(-\triangle)^{\sigma} v=0$ in a convex open domain $\Omega$,

- $v(x)$ is Lipschitz and bounded in $\mathbb{R}^{n} \backslash \Omega$.

Then $v \in C^{\sigma}\left(\mathbb{R}^{n}\right)$.

PROOF: Without loss of generality, we can assume that the Lipschitz constant of $v$ is 1 and $\|v\|_{L^{\infty}}=1$. Let $x_{0} \in \mathbb{R}^{n} \backslash \Omega$. Since $\Omega$ is convex, there is a unitary matrix $U$ such that the function $V(x)=\tilde{A}\left(U\left(x-x_{0}\right)\right)+v\left(x_{0}\right)$ satisfies $(-\triangle)^{\sigma} V=$ 0 in $\Omega$. Since $V(x) \geq \min \left(1,\left|x-x_{0}\right|\right)+v\left(x_{0}\right)$, then $V(x) \geq v(x)$ in $\mathbb{R}^{n} \backslash \Omega$. By the maximum principle $V \geq v$ in the whole $\mathbb{R}^{n}$. In the same way we prove $v(x) \geq v\left(x_{0}\right)-\tilde{A}\left(U\left(x-x_{0}\right)\right)$. Therefore we have a uniform $C^{\sigma}$-modulus of continuity for every point $x_{0}$ in $\mathbb{R}^{n} \backslash \Omega$. By Lemma $4.9, v \in C^{\sigma}\left(\mathbb{R}^{n}\right)$. 


\subsection{Optimal Regularity Results}

Optimal regularity can be quickly derived from what we have so far in the case when the contact set $\{u=0\}$ is convex. The nonconvex case will require more work.

THEOREM 5.2 Let $u$ be as in Theorem 4.12; if the interior of the contact set $\{x$ : $u(x)=0\}$ is convex, then $w \in C^{1-s}$ and therefore $u \in C^{1, s}$.

Proof: By Theorem 4.12, $w \in C^{\alpha}$ for some small $\alpha$. Then $w$ is continuous is $\mathbb{R}^{n}$. Let $\Omega$ be the interior of $\{x: u(x)=0\}$, that is, convex. We also know $w(x)=\phi(x)$ for every $x \in \mathbb{R}^{n} \backslash \Omega$, and $\phi$ is Lipschitz. Since $(-\triangle)^{1-s} w=0$ in $\Omega$, then we meet the conditions of Proposition 5.1 with $\sigma=1-s$, so we conclude $w \in C^{1-s}$.

Remark 5.3. With a slightly different barrier function it could be shown that in the situation of Theorem 4.16, $w \in C^{\alpha}$ for $\alpha=\min (1-s, \tau)$ where $\phi \in C^{\tau}$.

Remark 5.4. By constructing test functions that solve the equation outside of a ball instead of a semispace, the above theorem could be refined to a contact set that satisfies an exterior ball condition. For the time being we cannot assure any regularity for the free boundary, and such regularity theory is likely to require a sharp estimate on the regularity of the solution $u$.

The proofs from now on are not very different whether we consider $\phi$ to be Lipschitz or merely $C^{\tau}$. We will thus describe the general case right away. We suppose that in our original obstacle problem $\varphi \in C^{1, \beta}$ and $2 s<1+\beta$, so that we have $\phi \in C^{\tau}$ for $\tau=1+\beta-2 s$.

The following lemma gives us an idea about how far from convex the level sets of $u$ can be.

LeMMA 5.5 Let $u$ be as in Theorem 4.16. Let's assume that $w \in C^{\alpha}$ for some given $\alpha<1+\beta-2$ s (probably larger than the one from Theorem 4.16). Let $x_{0}$ be a point such that $u\left(x_{0}\right)=0$. Then for a small enough $\delta$, there is a constant $C_{0}$ such that $x_{0}$ is not in the convex envelope of the set

$$
A_{r}=\left\{x \in B_{r}\left(x_{0}\right): w(x)>w\left(x_{0}\right)+C_{0} r^{\alpha+\delta}\right\}
$$

for any $r>0$. Moreover, $\delta$ can be chosen to be any positive real number less than $((1+\beta) /(\alpha+2 s)-1) \alpha$ (for example, half of that number $)$.

Proof: Since $w \in C^{\alpha}$, then $u \in C^{\alpha+2 s}$ (or $C^{1, \alpha+2 s-1}$ ) so $w(x)=(-\triangle)^{s} u(x)$ can be computed by its integral representation.

We can assume $x_{0}=0$ without loss of generality. We choose

$$
\delta<\frac{1+\beta}{\alpha+2 s}-1 \alpha
$$

Notice that $\delta<\tau-\alpha$ (recall $\tau=1+\beta-2 s$ ). If we take $C_{0}=1$ in the definition of $A_{r}$, we will prove that the result of the lemma is true for $r$ small enough. Choosing 
$C_{0}$ large enough in (5.3), we can then make it true for larger values of $r$. (Actually, we can even make sure that $A_{r}$ is empty for large $r$.) So we will consider now

$$
A_{r}=\left\{x \in B_{r}\left(x_{0}\right): w(x)>w\left(x_{0}\right)+C_{0} r^{\alpha+\delta}\right\},
$$

and we want to show that for small enough $r, 0$ is not in the convex envelope of $A_{r}$.

Since $\phi$ is a $C^{\tau}$-function, $\phi(x) \leq \phi(0)+C|x|^{\tau}$. Then, when $x \in A_{r}$ for $r$ small enough,

$$
\phi(x) \leq \phi(0)+C|x|^{\tau} \leq w(0)+C_{0} r^{\alpha+\delta}<w(x) ;
$$

then $w(x)>\phi(x)$; thus $u(x)=0$ for every $x \in A_{r}$.

Let us argue by contradiction. Suppose that we have $k$ points $x_{1}, \ldots, x_{k} \in A_{r}$ such that a convex combination of them is 0 ,

$$
\sum_{j=1}^{k} \lambda_{j} x_{j}=0
$$

for $\lambda_{j} \geq 0$ and $\sum \lambda_{j}=1$. For each $j$, we have

$$
w\left(x_{j}\right)=\int_{\mathbb{R}^{n}} \frac{u\left(x_{j}\right)-u\left(x_{j}+y\right)}{|y|^{n+2 s}} \mathrm{~d} y=\int_{\mathbb{R}^{n}} \frac{-u\left(x_{j}+y\right)}{|y|^{n+2 s}} \mathrm{~d} y .
$$

Since $u$ satisfies (4.33), at each point $y$ there is a plane tangent from below with an error of order $C|z-y|^{1+\beta}$; i.e., there is a vector $A \in \mathbb{R}^{n}$ such that

$$
u(z) \geq u(y)+A \cdot(z-y)-C|z-y|^{1+\beta}
$$

for every $z \in \mathbb{R}^{n}$. If we replace $z=x_{j}+y$ in (5.5) and add, we get

$$
\sum_{j=1}^{k} \lambda_{j} u\left(x_{j}+y\right) \geq u(y)-C r^{1+\beta} .
$$

Now we compute $w(0)$ and compare it with the values of $w\left(x_{j}\right)$,

$$
\begin{aligned}
w(0) & =\int_{\mathbb{R}^{n}} \frac{u(0)-u(y)}{|y|^{n+2 s}} \mathrm{~d} y=\int_{\mathbb{R}^{n}} \frac{-u(y)}{|y|^{n+2 s}} \mathrm{~d} y \\
& =\int_{B_{\tilde{r}}} \frac{-u(y)}{|y|^{n+2 s}} \mathrm{~d} y+\int_{\mathbb{R}^{n} \backslash B_{\tilde{r}}} \frac{-u(y)}{|y|^{n+2 s}} \mathrm{~d} y .
\end{aligned}
$$

For the first term we use that $w \in C^{\alpha}$ and then $u \in C^{\alpha+2 s}$ (or $C^{1, \alpha+2 s-1}$ ). Then $u(x) \leq C|x|^{\alpha+2 s}$, and

$$
\int_{B_{\tilde{r}}} \frac{-u(y)}{|y|^{n+2 s}} \mathrm{~d} y \geq-C \tilde{r}^{\alpha}
$$


For the second term, we use (5.6):

$$
\begin{aligned}
\int_{\mathbb{R}^{n} \backslash B_{\tilde{r}}} \frac{-u(y)}{|y|^{n+2 s}} \mathrm{~d} y & \geq \int_{\mathbb{R}^{n} \backslash B_{\tilde{r}}} \frac{-\sum_{j=1}^{k} \lambda_{j} u\left(x_{j}+y\right)-C r^{1+\beta}}{|y|^{n+2 s}} \mathrm{~d} y \\
& \geq \int_{\mathbb{R}^{n} \backslash B_{\tilde{r}}} \frac{-\sum_{j=1}^{k} \lambda_{j} u\left(x_{j}+y\right)}{|y|^{n+2 s}} \mathrm{~d} y-C r^{1+\beta} \tilde{r}^{-2 s} .
\end{aligned}
$$

Now we make a convenient choice for $\tilde{r}$. Let $\tilde{r}=r^{p}$ for $p=(1+\beta) /(\alpha+2 s)$. We observe that since $p>(\alpha+\delta) / \alpha, x_{j} \in A_{r}$, and $u$ is $C^{\alpha}$, then for $r$ small enough $u\left(x_{j}+y\right)=0$ for every $y \in B_{\tilde{r}}$. Thus

$$
\begin{aligned}
\int_{\mathbb{R}^{n} \backslash B_{\tilde{r}}} \frac{-u(y)}{|y|^{n+2 s}} \mathrm{~d} y & \geq \int_{\mathbb{R}^{n} \backslash B_{\tilde{r}}} \frac{-\sum_{j=1}^{k} \lambda_{j} u\left(x_{j}+y\right)}{|y|^{n+2 s}} \mathrm{~d} y-C r^{1+\beta} \tilde{r}^{-2 s} \\
& \geq \sum_{j=1}^{k} \lambda_{j} w\left(x_{j}\right)-C r^{1+\beta} \tilde{r}^{-2 s} \\
& \geq w(0)+C{ }_{0} r^{\alpha+\delta}-C r^{1+\beta-2 s p}
\end{aligned}
$$

Adding (5.7) to (5.8), we get

$$
\begin{aligned}
w(0) & \geq-C \tilde{r}^{\alpha}+w(0)+C_{0} r^{\alpha+\delta}-C r^{1+\beta-2 s p} \\
& \geq w(0)+C_{0} r^{\alpha+\delta}-C r^{p \alpha}-C r^{1+\beta-2 s p} \\
& \geq w(0)+C_{0} r^{\alpha+\delta}-C r^{\frac{(1+\beta) \alpha}{\alpha+2 s}} .
\end{aligned}
$$

But this is impossible for small $r$ because we chose $\delta$ so that $\alpha+\delta<2 \alpha /(\alpha+2 s)$.

LEMMA 5.6 Let $u$ be as in Theorem 4.16. Let's assume that $w \in C^{\alpha}$ for some given $\alpha<\min (1-s, 1+\beta-2 s$ ) (probably larger than the one from Theorem 4.16). Then $w$ is actually in $C^{\gamma \alpha}$, where

$$
\begin{aligned}
\gamma & =\frac{1-s}{1-s+\delta} \cdot \frac{\alpha+\delta}{\alpha} \\
& =\left(\frac{(1-s)(\alpha+2 s+1+\beta)}{2(1-s)(\alpha+2 s)+\alpha(1+\beta-\alpha-2 s)}\right)>1
\end{aligned}
$$

where $\delta$ is as defined in Lemma 5.5.

PROOF: First we will construct some auxiliary functions. Let $B$ be as in (5.1) with $\sigma=1-s$. Recall that $B(x) \geq \beta$ when $|x| \geq 1$. Then, for $1>r>0$, let

$$
D(x)=\frac{r^{\alpha}}{d_{1}} B\left(\frac{x}{r}\right)+\int_{r}^{1} \frac{1}{d_{1}} B\left(\frac{x}{t}\right) t^{\alpha-1} \mathrm{~d} t .
$$


Clearly, $D(x)=0$ when $|x|<r$ and $x_{n} \geq 0$. When $|x| \geq r$,

$$
D(x) \geq \frac{r^{\alpha}}{d_{1}} d_{1}+\int_{r}^{\min (|x|, 1)} \frac{1}{\alpha d_{1}} d_{1} t^{\alpha-1} \mathrm{~d} t \geq \min \left(|x|^{\alpha}, 1\right) .
$$

For $x_{n}<0$ and $|x|<r / 2$, applying (5.2), we have

$$
\begin{aligned}
D(x) & \leq C\left(r^{\alpha}\left(\frac{\left|x_{n}\right|}{r}\right)^{1-s}+\int_{r}^{1}\left(\frac{\left|x_{n}\right|}{t}\right)^{1-s} t^{\alpha+1} \mathrm{~d} t\right) \\
& \leq C\left(r^{\alpha+s-1}+1\right)\left|x_{n}\right|^{1-s} .
\end{aligned}
$$

Now, let us take a point in the free boundary $\partial\{u=0\}$ that we will suppose to be the origin. Since $w$ is $C^{\alpha}$ for $0<r<1$, by (5.10) there is a constant $C$ such that $w(x)-w(0) \leq C D(x)$ for every $|x|>r$. By Lemma 5.5, if we choose $r$ small enough, then $w(x) \leq w(0)+r^{\alpha+\delta}$ at least in half of the ball $B_{r}$. We can assume that $B_{r} \cap\left\{x_{n} \geq 0\right\}$ is that half of the ball. Therefore

$$
w(x) \leq w(0)+r^{\alpha+\delta}+C D(x)
$$

for every $x$ except maybe some $x \in B_{r} \cap\left\{x_{n}<0\right\}$.

Since $\phi$ is $C^{\tau}$ and $r$ was chosen small, $\phi(x) \leq \phi(0)+C|x|^{\tau}=w(0)+C|x|^{\tau} \leq$ $w(0)+r^{\alpha+\delta}+C D(x)$. Therefore all the points for which (5.12) does not hold must be in the set where $w(x)>\phi(x)$, i.e., in the interior of $\{u(x)=0\}$. But in that set $(-\triangle)^{1-s} w=0$; also $(-\triangle)^{1-s} D=0$ in that set (since it is included in $\left.\left\{x_{n}<0\right\} \cap B_{r}\right)$. By the maximum principle, (5.12) holds in all of $\mathbb{R}^{n}$.

Let $x$ be such that $|x|$ is small. Let $p=(1-s) /(1-s+\delta)<1$ and $r=$ $|x|^{p}>2|x|$. Then, combining (5.12) with (5.11), we get

$$
\begin{aligned}
w(x) & \leq w(0)+r^{\alpha+\delta}+C\left(r^{\alpha+s-1}+1\right)\left|x_{n}\right|^{1-s} \\
& \leq w(0)+|x|^{(\alpha+\delta) p}+C\left(|x|^{(\alpha+s-1) p}+1\right)|x|^{1-s} \\
& \leq w(0)+C\left(|x|^{q}+|x|^{1-s}\right) \leq w(0)+C|x|^{q},
\end{aligned}
$$

where

$$
\begin{aligned}
q=(\alpha+\delta) p=\frac{(\alpha+\delta)(1-s)}{1-s+\delta} & =\frac{(\alpha+s-1)(1-s)}{1-s+\delta} \\
& =(\alpha+s-1) p+(1-s)<1-s .
\end{aligned}
$$

Since $w(0)=\phi(0)$, then $w(x) \geq \phi(x) \geq \phi(0)-C|x| \geq \phi(0)-C|x|^{q}$ for $|x|$ small. And this $C^{q}$ modulus of continuity holds at every point in the free boundary $\partial\{u=0\}$.

Let $x_{0}$ be such that $u\left(x_{0}\right)>0$; let $x$ be any other point in $\mathbb{R}^{n}$. Let $x_{1}$ be a point in the segment between $x$ and $x_{0}$ that is in the free boundary $\partial\{u=0\}$. Then

$$
\begin{aligned}
\left|w\left(x_{0}\right)-w(x)\right| & \leq\left|w\left(x_{0}\right)-w\left(x_{1}\right)\right|+\left|w\left(x_{1}\right)-w(x)\right| \\
& \leq\left|\phi\left(x_{0}\right)-\phi\left(x_{1}\right)\right|+\left|w\left(x_{1}\right)-w(x)\right| \\
& \leq C\left(\left|x_{0}-x_{1}\right|^{\tau}+\left|x_{1}-x\right|^{q}\right) \leq C\left|x_{0}-x\right|^{q} .
\end{aligned}
$$


Thus, there is a uniform $C^{q}$ modulus of continuity for every $x$ in the set $\overline{\{u>0\}}$. Since $(-\triangle)^{1-s} w=0$ in the complement of this set, we can apply Lemma 4.15 to conclude $w \in C^{q}\left(\mathbb{R}^{n}\right)$. Recalling that in Lemma $5.5 \delta$ can be chosen to be

$$
\frac{1}{2}\left(\frac{1+\beta}{\alpha+2 s}-1\right) \alpha
$$

we get a complicated formula for $q$,

$$
\begin{aligned}
q & =\frac{(\alpha+\delta)(1-s)}{1-s+\delta}=\frac{1-s}{1-s+\delta} \cdot \frac{\alpha+\delta}{\alpha} \cdot \alpha \\
& =\left(\frac{(1-s)(\alpha+2 s+1+\beta)}{2(1-s)(\alpha+2 s)+\alpha(1+\beta-\alpha-2 s)}\right) \alpha \\
& =\gamma \alpha .
\end{aligned}
$$

Proposition 5.7 Let $u$ and $w$ be as in Theorem 4.16. Then $w \in C^{\alpha}$ for every $\alpha<\min (1-s, 1+\beta-2 s)$. Thus $u \in C^{1, \alpha}$ for every $\alpha<\min (s, \beta)$.

PROOF: From Theorem 4.12, we know that $w \in C^{\alpha}$ for some small $\alpha>0$. Then we can apply Lemma 5.6 repeatedly to get $w \in C^{\alpha}$ for larger values of $\alpha$. To check that $\alpha$ gets as close to $\min (1-s, 1+\beta-2 s)$ as desired, we only have to observe that the application $I(\alpha)=\gamma \alpha$, where $\gamma$ is given by (5.9), is continuous and such that $I(\alpha)>\alpha$ for every $\alpha \in(0, \min (1-s, 1+\beta-2 s))$ and $I(\min (1-s, 1+\beta-2 s))=\min (1-s, 1+\beta-2 s)$.

THEOREM 5.8 Let $\beta>0$. Given a function $\varphi \in C^{1, \beta}$, let $u$ be the solution of the obstacle problem given by (1.1)-(1.4). Then $u \in C^{1, \alpha}$ for every positive number $\alpha$ less than $\min (\beta, s)$.

Proof: In the case $1+\beta>2 s$, we apply Proposition 5.7 to $u-\varphi$ with $\phi=-(-\triangle)^{s} \varphi$. Recall that $u-\varphi$ satisfies (4.33) and (4.34) because of Proposition 3.11 and Proposition 3.13, and (4.7) is satisfied because of Corollary 3.12.

In the case $1+\beta \leq 2 s$, the proof is simpler. From the definition of the problem (1.1)-(1.4) (or from Proposition 3.1 if we start with the variational approach) $(-\triangle)^{s} u \geq 0$ in $\mathbb{R}^{n}$. Moreover, $(-\triangle)^{\sigma} u \geq 0$ in $\mathbb{R}^{n}$ for any $\sigma \leq s$, since $(-\triangle)^{\sigma} u=(-\triangle)^{\sigma-s}(-\triangle)^{s} u$, and the operator $(-\triangle)^{\sigma-s}$ is given by the convolution with a positive kernel. Since $\varphi \in C^{1+\beta},(-\triangle)^{\sigma} \varphi \in L^{\infty}$ for any $\sigma<(1+\beta) / 2$. Then we can apply Proposition 3.11 to conclude that $(-\triangle)^{\sigma} u \leq C$ in $\mathbb{R}^{n}$ for any $\sigma<(1+\beta) / 2$. Thus $(-\triangle)^{\sigma} u \in L^{\infty}$ for any $\sigma<(1+\beta) / 2$, and from Proposition $2.9 u \in C^{1, \alpha}$ for any $\alpha<\beta$.

Acknowledgment. This work is part of my doctoral thesis. I would like to thank my advisor Prof. Luis Caffarelli for suggesting the problem and for all the useful discussion and encouragement. 
I would also like to thank Alex Kahle and Russell Schwab for helping with the proofreading of this manuscript.

\section{Bibliography}

[1] Athanasopoulos, I.; Caffarelli, L. A. Optimal regularity of lower dimensional obstacle problems. Preprint.

[2] Boyarchenko, S. I.; Levendorskiǔ, S. Z. Perpetual American options under Lévy processes. SIAM J. Control Optim. 40 (2002), no. 6, 1663-1696 (electronic).

[3] Caffarelli, L. A. Further regularity for the Signorini problem. Comm. Partial Differential Equations 4 (1979), no. 9, 1067-1075.

[4] Caffarelli, L. A. The obstacle problem revisited. J. Fourier Anal. Appl. 4 (1998), no. 4-5, $383-$ 402.

[5] Caffarelli, L. A.; Kinderlehrer, D. Potential methods in variational inequalities. J. Analyse Math. 37 (1980), 285-295.

[6] Cont, R.; Tankov, P. Financial modelling with jump processes. Chapman \& Hall/CRC Financial Mathematics Series. Chapman \& Hall/CRC, Boca Raton, Fla., 2004.

[7] Frehse, J. On the regularity of the solution of a second order variational inequality. Boll. Un. Mat. Ital. (4) 6 (1972), 312-315.

[8] Gilbarg, D.; Trudinger, N. S. Elliptic partial differential equations of second order. Reprint of the 1998 edition. Classics in Mathematics. Springer, Berlin, 2001.

[9] Landkof, N. S. Foundations of modern potential theory. Die Grundlehren der mathematischen Wissenschaften, Band 180. Translated from the Russian by A. P. Doohovskoy. Springer, New York, 1972.

[10] Levendorskiū, S. Z. Pricing of the American put under Lévy processes. Int. J. Theor. Appl. Finance 7 (2004), no. 3, 303-335.

[11] Pham, H. Optimal stopping, free boundary, and American option in a jump-diffusion model. Appl. Math. Optim. 35 (1997), no. 2, 145-164.

[12] Richardson, D. Doctoral dissertation. University of British Columbia, Vancouver, 1978.

[13] Silvestre, L. E. Hölder estimates for solutions of integro differential equations like the fractional Laplace. Preprint.

[14] Stein, E. M. Singular integrals and differentiability properties of functions. Princeton Mathematical Series, No. 30. Princeton University Press, Princeton, N.J., 1970.

LUIS SILVESTRE

Courant Institute

251 Mercer Street

New York, NY 10012

E-mail: silvestrecims.nyu.edu

Received March 2005. 